\title{
Genome-wide characterization and expression analysis of soybean trihelix gene family
}

\author{
Wei Liu ${ }^{1,2}$, Yanwei Zhang ${ }^{1,2}$, Wei $\mathbf{L i}^{1,2}$, Yanhui Lin ${ }^{3}$, Caijie Wang ${ }^{1,2}$, Ran Xu ${ }^{1,2}$, Lifeng Zhang ${ }^{\text {Corresp. 1,2 }}$ \\ ${ }^{1}$ Crop Research Institute $\square$ Shandong Academy of Agricultural Sciences, Jinan, Shandong \\ 2 Shandong Engineering Laboratory of Featured Crops, Jinan, China \\ 3 Institute of Food Crops, Hainan Academy of Agricultural Sciences, Haikou, China \\ Corresponding Author: Lifeng Zhang \\ Email address: zhanglifeng9639@sina.com
}

Trihelix transcription factors play multiple roles in plant growth, development and various stress responses. In this study, we identified 71 trihelix family genes in the soybean genome. These trihelix genes were located at 19 out of 20 soybean chromosomes unevenly and were classified into six distinct subfamilies: GT-1, GT-2, GTY, SIP1, SH4 and GTס. The gene structure and conserved functional domain of these trihelix genes were similar in the same subfamily but diverged between different subfamilies. 13 segmental duplicated gene pairs were identified and all of them experienced a strong purifying selective pressure during evolution. Various stress-responsive cis-elements presented in the promoters of soybean trihelix genes, suggesting that the trihelix genes might respond to the environmental stresses in soybean. The expression analysis suggests that trihelix genes are involved in diverse functions during soybean development, flood or salinity tolerance, and plant immunity. Our results provide comprehensive genomic information of the soybean trihelix genes and a basis for further characterizing their roles in response to environmental stresses. 
1 Genome-wide characterization and expression analysis of soybean trihelix gene family

2 Wei Liu ${ }^{1,2}$, Yanwei Zhang ${ }^{1,2}$, Wei Li ${ }^{1,2}$, Yanhui $\operatorname{Lin}^{3}$, Caijie Wang ${ }^{1,2}$, Ran $\mathrm{Xu}^{1,2}$, Lifeng

3 Zhang ${ }^{1,2^{*}}$

$4{ }^{1}$ Crop Research Institute, Shandong Academy of Agricultural Sciences, Jinan, Shandong, China

$5 \quad{ }^{2}$ Shandong Engineering Laboratory of Featured Crops, Jinan, Shandong, China.

6 Institute of Food Crops, Hainan Academy of Agricultural Sciences, Haikou, Hainan, China.

7 *Corresponding Author:

8 Lifeng Zhang ${ }^{1}$

9202 Gongye North Road, Jinan, Shandong, 250100, China

10 Email address: zhanglifeng9639@sina.com

11 Abstract

12 Trihelix transcription factors play multiple roles in plant growth, development and various stress responses. In this study, we identified 71 trihelix family genes in the soybean genome. These

14 trihelix genes were located at 19 out of 20 soybean chromosomes unevenly and were classified 15 into six distinct subfamilies: GT-1, GT-2, GT $\gamma$, SIP1, SH4 and GT $\delta$. The gene structure and conserved functional domain of these trihelix genes were similar in the same subfamily but 17 diverged between different subfamilies. 13 segmental duplicated gene pairs were identified and 18 all of them experienced a strong purifying selective pressure during evolution. Various stressresponsive cis-elements presented in the promoters of soybean trihelix genes, suggesting that the trihelix genes might respond to the environmental stresses in soybean. The expression analysis

21 suggests that trihelix genes are involved in diverse functions during soybean development, flood 22 or salinity tolerance, and plant immunity. Our results provide comprehensive genomic 23 information of the soybean trihelix genes and a basis for further characterizing their roles in 24 response to environmental stresses. 
26

27

28

29

30

31

32

33

34

35

36

37

38

39

40

41

42

43

44

45

46

47

48

49

50

51

52

53

54

55

56

\section{Introduction}

Transcription factors (TFs) are a type of DNA binding protein. They are capable of interacting with the cis-element of the promoter regions of the target genes, and regulate their expression. TFs play important roles in multiple development process and stress response in plants. Of all the reported TFs, the trihelix family is one of the first TFs discovered in plants (Green et al.,1987).

The trihelix family genes share one or two trihelix (helix-loop-helix-loop-helix) structures, each consisting of three putative-helices, which are responsible for binding to the GT motif (a light-responsive DNA element) (Zhou et al., 1999). Initial study of this family focused on the regulation of light-responsive genes (Green et al., 1987; Zhou et al., 1999). Subsequently, a majority of trihelix genes belonging to the different subfamilies have been cloned and characterized. Those trihelix genes were found to be involved in multiple development and growth processes such as: flowering, stomatal development, embryogenesis, and seed development (Tzafrir et al., 2004; Breuer et al., 2009; Gao et al., 2009; Barr et al., 2012; Qin et al., 2014). With the in-depth study of this gene family, more and more trihelix genes were proven to play important roles in abiotic and biotic stress resistance. The 3 rice (Oryza sativa L.) trihelix genes $(O s G T-\gamma-1, O s G T \gamma-2$ and $O s G T \gamma-3)$ and two soybean (Glycine max (L.) Merr.) trihelix genes ( $G m G T-2 A$ and $G m G T-2 B)$ were reported to be related to cold, drought, and salt stress response (Xie et al., 2009; Fang et al., 2010). The expression of Arabidopsis (Arabidopsis thaliana) trihelix gene, AtGT2L, could be induced by cold and salt stresses (Xi et al., 2012); GT2 LIKE 1 (GTL1) loss-of-function mutations result in increased water deficit tolerance in Arabidopsis (Yoo et al., 2010). An Oilseed rape (Brassica napus) gene BnSIP1-1 played roles in ABA synthesis and signaling, as well as salt and osmotic stress response (Luo et al., 2017). In addition, it was also reported that the trihelix genes respond to waterlogging stress in maize (Zea mays L.) (Du et al., 2016). Meanwhile, the Arabidopsis gene ASR3 could regulate the expression of genes related to immunity (Li et al., 2015).

As an important leguminous crop in the world, soybean provides an essential source of protein to the human diet, feed for live-stock, and as feedstock for the bio-diesel industry (Wilcox., 2004; Koberg et al., 2011). However, soybean production can be dramatically decreased by the occurrence of environmental stresses, such as flooding (waterlogging or submergence) (Ahmed et al., 2012), high-salt (Wang et al., 1999), as well as pathogen infection (Tyler., 2007). Several reports had performed the transcriptome analysis of soybean under abiotic 
57 and biotic stress (Nanjo et al., 2011; Belamkar et al., 2014; Chen et al., 2016; Yin et al., 2016).

58 With the completion of soybean genome sequencing, many TF families including the 59 abiotic/biotic stress-responsive transcription factor families have been genome-wide analyzed

60 (Song et al., 2018, Yu et al., 2016, Wang et al., 2019). Considering the potential roles of trihelix

61 family genes in stress tolerance, genome-wide analysis of soybean trihelix genes is necessary to

62 be studied in depth and comprehensively.

63 In this study, we identified the soybean trihelix genes by the Myb/SANT-LIKE domain using

64 HMM (Hidden Markow Model)-searches against a new version of the reference soybean genome

65 (Wm82.a2. v1). We next comprehensively analyzed their chromosomal distributions, gene

66 duplication, motif composition, gene structure, cis-elements, phosphorylation sites, and miRNA

67 targeting. Subsequently, we performed public-RNA-Seq data analysis to explore their tissue

68

69

70

71

72

73

74

75

76

77

78

79

80

81

82

83

84

85

expression patterns. Furthermore, by analyzing the expression profile of trihelix genes in soybean suffering from submergence, high-salt and MAMP (microbe-associated molecular patterns) mixture treatment at the seedling stage, we identified several candidate trihelix genes that might contribute to the stress tolerance in soybean. Our results provide more comprehensive information of soybean trihelix genes and theoretical basis for their functional analysis, especially in the environmental stress responses.

Materials \& Methods

Genome-wide identification of trihelix TFs in soybean

The genome sequences of soybean were downloaded from the Phytozome database (Goodstein et al., 2011) (v.12.1) (https://phytozome.jgi.doe.gov/pz/portal.html). The Hidden Markow Model (HMM) of Myb/SANT-LIKE domain (PF13837) was retrieved from the Pfam database (http://pfam.xfam.org) (Finn et al., 2015). To identify candidate trihelix TFs, HMMER program was used to search the soybean genome with an E-value cutoff $<\mathrm{e}^{-5}$. All putative proteins were confirmed by the Pfam and NCBI-CDD database (https://www.ncbi.nlm.nih.gov/cdd). The molecular weights and isoelectric points of soybean trihelix proteins were estimated using the online software ExPASy (https://www.expasy.org) (Artimo et al., 2012). Subcellular localization was predicted by using CELLO (V.2.5, http://cello.life.nctu.edu.tw/) (Yu et al., 2006).

\section{Multiple alignment and phylogenetic analysis}

Peer) reviewing PDF | (2019:08:40490:3:0:NEW 29 Jan 2020) 
87 Multiple sequence alignments were performed using Clustalw with the amino acid sequences of 88 trihelix family proteins. A neighbor-joining (NJ) phylogenetic tree was constructed using MEGA 89 (v.7.0) (https://www.megasoftware.net) (Kumar et al., 2016) by the method with the following 90 parameters: Poisson correction, pairwise deletion, and 1000 bootstrap replicates.

91

92 Gene structure analysis and identification of conserved motifs

93 The gene structures of trihelix genes were determined by the Gene Structure Display Server 94 (http://gsds.cbi.pku.edu.cn) (Hu et al., 2014) using the coding and genomic sequences of the 95 96 soybean trihelix family genes. The MEME program (http://memesuite.org/tools/meme) (Bailey et al., 2015) was used to identify conserved motifs among all the soybean trihelix genes with using default parameters except for the flowering parameter: the maximum number of motifs, 10 .

Gene duplication and Ks calculate

100 The chromosomal location map was constructed by using the MapChart (v.2.32) program 101 (Voorrips et al., 2002). Major criteria was used for analyzing potential gene duplications; 102 Potential gene duplications were determined by two major criteria: length of aligned sequence 103 covers $\geq 75 \%$ of longer gene and similarity of aligned regions is $\geq 75 \%$. Ka and Ks values were 104 calculated using KaKs Calculator (http://code.google.com/p/kaks calculator/wiki/KaKs_Calculator) (Zhang et al., 2006).

106

107

108

109

110

111

112

113

114

115

116

117

\section{Cis-Element analysis of trihelix TF family}

The $1500 \mathrm{bp}$ upstream sequences of 71 soybean trihelix family genes were obtained from Phytozome database and identified as a hypothetical promoter. The PLANTCARE database (http://bioinformatics.psb.ugent.be/webtools/plantcare/html/) was used to analyze the cisregulatory elements of the trihelix family gene promoters.

\section{Mitogen-activated protein kinase (MAPK)-specific phosphorylation sites and miRNA} target prediction

MAPK-specific phosphorylation sites were predicted by the Musite (version 1.0)

(http://musite.net) tool at the specificity level of $\geq 98 \%$ (Gao et al. 2010). The online sever psRNA Target (http://plantgrn.noble.org/psRNATarget/, Dai et al., 2018) was used to predict 
118 miRNA targets with using the parameters as follows: maximum expectation of 3 and maximum 119 energy to unpair target site (UPE) of less than 25.

120

\section{Expression analysis of soybean trihelix genes in different tissues using RNA-Seq Atlas}

122 To analyze expression pattern associated with gene function, 71 soybean trihelix family genes

123

124

125

126

127

128

129

130

131

132

133

134

135

136

137

138

139

140

141

142

143

144

145

146

147

148 were investigated based on the public RNA-seq data published in Phytozome v12.1 (Libault et al., 2010; Goodstein et al., 2011). The expression of the 71 soybean trihelix family genes were analyzed from 9 different soybean tissues, including flowers, leaves, nodules, pods, roots, root hairs, seeds, shoot apical meristems (SAMs) and stems (Libault et al., 2010).

\section{Analysis of differentially expressed soybean trihelix genes in response to the submergence and MAMP mixture treatment}

For the analysis of trihelix genes in response to the submergence stress at the seedling stage, the FPKM (Fragments per kilobase for a million reads) of all the soybean trihelix genes was obtained from our previous transcriptome data of soybean (14-day-old seedlings) at $3 \mathrm{~h}, 6 \mathrm{~h}, 12 \mathrm{~h}$ and $24 \mathrm{~h}$ after submergence (Lin et al., 2019). The raw RNA-seq data were deposited in the NCBI Sequence Read Archive (SRA), and the accession number is SRP181976. The soybean trihelix genes that showed more than two-fold expression changes $(\mid \log 2$ (Fold Change) $\mid \geq 1)$ with the adjusted $P$ value of less than 0.05 were considered as differentially expressed genes (DEGs). For the analysis of trihelix genes in response to the MAMP mixture treatment, publicly available microarray data (series accession no. GSE32642) was obtained from the Gene Expression Omnibus (GEO) database (https://www.ncbi.nlm.nih.gov/geo/), where the soybean leaves were treated by the MAMP mixture (flagellin 22 (flg22) and crab shell chitin) (Valdés-López et al., 2011). The microarray data was processed using the NCBI GEO software, GEO2R

(https://www.ncbi.nlm.nih.gov/geo/geo2r/). The soybean trihelix genes that showed more than two-fold expression changes $(\mid \log 2$ (Fold Change) $\mid \geq 1)$ with the adjusted $P$ value of less than 0.05 were considered as differentially expressed genes (DEGs).

\section{Plant growth conditions and treatments}

The seedlings of a soybean cultivar Qihuang 34 were grown in a controlled culture room at $25^{\circ} \mathrm{C}$ under long day (16/8 h light/dark) condition. For the submergence treatment, the seedlings (14- 
149 day-old) grown in the pots were completely immersed in water by placing them in the white 150 plastic containers filled with water. The whole roots of treated soybean were sampled at $3 \mathrm{~h}, 6 \mathrm{~h}$, $151 \quad 12 \mathrm{~h}$ and $24 \mathrm{~h}$ time point and immediately placed in liquid nitrogen and stored at $-80{ }^{\circ} \mathrm{C}$ until 152 RNA extraction for quantitative real-time PCR (qRT-PCR). All root material from 10 plants was 153 pooled and three biological replicates were performed.

154 For the $\mathrm{NaCl}$ and $\mathrm{ABA}$ treatments, 14-day-old seedlings were removed from the soil and 155 cultured in Hoagland liquid medium for 2 days, and then transferred into new Hoagland liquid 156 medium containing $100 \mathrm{mM} \mathrm{NaCl}$ or $100 \mu \mathrm{M}$ ABA, respectively. The whole roots of control and 157 treated seedlings were sampled at $0 \mathrm{~h}, 3 \mathrm{~h}, 6 \mathrm{~h}$, and $12 \mathrm{~h}$ after treatment. All samples were 158 rapidly frozen in liquid nitrogen and stored at $-80{ }^{\circ} \mathrm{C}$. All root material from 5 plants was pooled 159 and three biological replicates were performed.

160 For the salicylic acid (SA), Jasmonic acid (JA) and ethylene precursor 1-aminocyclopropane-1161 carboxylicacid (ACC) treatment, 14-day-old seedlings were sprayed with 1mM SA, $100 \mu \mathrm{M} \mathrm{JA}$ 162 or $100 \mu \mathrm{M}$ ACC on the leaf, respectively. The leaves of control and treated seedlings were 163 sampled at $0 \mathrm{~h}, 30 \mathrm{~min}, 3 \mathrm{~h}, 6 \mathrm{~h}$, and $12 \mathrm{~h}$ after treatment. All samples were rapidly frozen in liquid nitrogen and stored at $-80^{\circ} \mathrm{C}$. Leaves from 5 plants were pooled and three biological replicates were performed.

166

167

168

169

170

171

172

173

174

175

176

177

178

179

\section{qRT-PCR analysis}

The total RNA from all of the roots or leaves of soybean plants suffering from submergence $/ \mathrm{NaCl}$ stress or hormone treatments (ABA, SA, JA and $\mathrm{ACC}$ ) and the control was isolated using Trizol reagent. qRT-PCR was performed using Roche 480 Light Cycler (Roche, Mannheim, Germany) using a Takara SYBR Premix Extaq (Takara, Japan). Three biological replicates were analyzed, with 3 technical replicates for each of the triplicate biological samples. The calculation of gene expression levels followed the $2^{-\Delta \Delta \mathrm{CT}}$ method described by Livak and Schmittgen (2001). GmActin (Glyma.18G290800) was used as the internal reference gene for the submergence, JA, SA and ACC treatment (Song et al., 2018), and GmELF1b (Glyma.02G276600) was used as the internal reference gene for the salinity and ABA treatments (Yim et al., 2015; Li et al., 2016). The primers of the selected trihelix genes used for qRT-PCR are listed in Table S9.

\section{Results}


180 Identification and duplication analysis of soybean trihelix genes

181 A total of 71 trihelix genes were identified in soybean genome. In addition to the 63 trihelix

182 genes reported previously, 8 new soybean trihelix genes (Glyma.04G135400,

183 Glyma.05G200400, Glyma.08G007800, Glyma.10G064900, Glyma.15G234100,

184 Glyma.17G194700, Glyma.20G184500 and Glyma.20G189000) were also been identified. The

185 characteristics of all these 71 soybean trihelix genes including gene Locus, chromosomes

186 locations, lengths of the CDS (coding sequence) and protein, protein sizes, isoelectric points and

187 subcellular localization were summarized in Table S1. The length of the CDS vary from 753 to

$1882667 \mathrm{bp}$, with predicted protein products varying in length from 250 (Glyma.15G082900) to 888

189 (Glyma.04G194600) amino acids. Their protein MW (Molecular Weight) range from $28.62 \mathrm{kDa}$

190 to $97.37 \mathrm{kDa}$. Their predicted isoelectric points vary from 4.52 (Glyma.07G087900) to 10.02

191 (Glyma.13G290500). Almost all of the trihelix proteins were predicted to have nuclear

192 localization except for Glyma.04G194600, Glyma.06G171400 and Glyma.10G201400 which

193 were localized to the chloroplast (Table S1).

194

195 These 71 soybean trihelix genes are distributed on 19 out of 20 chromosomes. Chromosome 10

196 (Chr 10) contain the largest number of trihelix genes (10 trihelix genes) whereas Chr5 and Chr12 197 contain only one. No trihelix genes were detected on Chr14 (Figure 1). We then investigated the 198 gene duplication events of the trihelix genes. No trihelix gene in soybean was identified as a 199 tandem duplication, and 13 pairs of genes were found to be segmental duplications (Figure 2, 200 Table 1). Simultaneously, the nonsynonymous (Ka) and synonymous (Ks) substitution rates 201 between these duplicated gene pairs were calculated. The Ka/Ks of all the 13 duplicated gene 202 pairs (Table 1) was found to be less than 1, suggesting that the soybean trihelix gene family 203 might have experienced a strong purifying selective pressure during evolution. Moreover, the 204 approximate date of duplication events was calculated using $\mathrm{T}=\mathrm{Ks} / 2 \lambda$ (Nei et al., 2000), 205 assuming that the divergence rate of 6.161029 synonymous mutations per synonymous site per 206 year for soybean (Lynch et al., 2000). The segmental duplications of the trihelix genes in 207 soybean originate from 1.67 million years ago $(\mathrm{Mya})(\mathrm{Ks}=0.02)$ to $19.80 \mathrm{Mya}(\mathrm{Ks}=0.24)$ 208 (Table 1), with the mean of $12.18 \mathrm{Mya}(\mathrm{Ks}=0.1487)$. 
210 Phylogenetic relationships, conserved protein motifs and gene structure analysis of soybean 211 trihelix genes

212 To uncover the classifications of the trihelix proteins of soybean, a phylogenetic analysis of the 213 amino acid sequences with the trihelix TFs from soybean and other species (Arabidopsis, rice 214 and tomato) was conducted. As shown in Figure 3, all of the trihelix genes are divided into six 215 subfamilies: SIP1, GT $\gamma$, GT $\delta$, GT-1, GT-2 and SH4. This classification is largely similar to the 216 previous analyses of trihelix TFs in Arabidopsis, rice and tomato (Kaplan-Levy et al., 2012; Yu et 217 al., 2015; Li et al., 2019). The 71 soybean trihelix genes are distributed over all of these 218 subfamilies. GT-2 and SIP1 are the largest subfamilies, both containing 22 soybean trihelix 219 genes. Whereas the GT $\delta$ subfamily is the smallest, only containing 3 members. This result 220 suggests that soybean trihelix genes are distributed unevenly in the different subfamilies (Figure $2213)$.

222 The DNA-binding domain of trihelix genes features a typical trihelix (helix-loop-helix223 loop-helix) structure. By performing the multiple sequence alignment, we found that GT binding 224 motifs were highly conserved among soybean and Arabidopsis trihelix genes (Figure S1). Four 225 amino acids of the tress tandem helices were found to be invariant in almost all of the trihelix 226 genes, including tryptophan (W) and leucine (L) in Helix1; tryptophan (W) in Helix2; and 227 cysteine (C) in Helix3 (Figure S1).

228

We then used the MEME program to detect conserved motifs in the soybean trihelix 230 family and found 10 distinct motifs (Figure 4A). Among them, 5 motifs are matched up with the 231 GT binding domains. Motif 2 is in Helix 1 domain. Motif 4 is Helix 1 and Helix 2, motif 10 is 232 Helix 2, motif 1 or motif 6 is Helix 3. The details of these 10 motifs are shown in Figure S2. In 233 general, genes from the same subfamily are characterized by a similar motif type and 234 distribution. Almost all of the SIP1 subfamily members (except Glyma15G082900, 235 Glyma.10G065100, Glyma.13G149900, Glyma.10G201400 and Glyma.20G189000) contain 236 Motif 4, 6 and 8. Moreover, the motif 8 is only identified in this subfamily. All of the SH4 237 subfamily members (expect Glyma.17G194700 and Glyma.20G184500) contain Motifs 2 and 6. 238 GT $\gamma$ subfamily contains motifs 1 and 7 (except Glyma.10G064900 contains motif 6 and 7). 239 Motif 7 is only present in the GT $\gamma$ subfamily. Both motif 2 and 10 are present in the GT-1and 
240 GT-2 subfamilies, and the GT-2 subfamily contains more motifs than any other subfamilies. All 241 of the 3 trihelix proteins belonging to GT $\delta$ subfamily contain motif 2, 6 and 10 (Figure 4A).

242 To identify the features of the soybean trihelix gene family, the structures of these trihelix 243 genes were further analyzed (Figure 4B). As shown in Figure 4B, the number of the soybean 244 trihelix gene exons is discontinuously distributed from 1 to 17 . The average number of exons is 245 the lowest in GT $\gamma$ subfamily, and highest in GT $\delta$ subfamily (Figure S3). We found that GT $\gamma$ 246 genes only had 1-2 exons. 17 out of 22 genes belonging to GT- 2 subfamily have 2 exons and 247 only 1 intron. In the SIP1 subfamily, 18 out of 22 members have 1-3 exons, and other 4 members 248 have 7 or 8 exons; members belong to the SH4 subfamily have 2-4 exons. In the GT-1 249 subfamily, 4 members (Glyma.01G121000, Glyma.07G151100, Glyma.18G202400 and 250 Glyma.01G150500) have 2 exons, and the other 3 members have 4-5 exons. 3 members 251 (Glyma.17G128500, Glyma.04G194600 and Glyma.06G171400) of the GT $\delta$ subfamily are the 252 largest genes with 17 exons. In general, members of the same family might share similar gene 253 structure.

254

255

\section{Expression profiling of trihelix genes in different tissues of soybean}

To investigate the transcript abundance of soybean trihelix genes, expression profiles covering 9 tissues in soybean were analyzed by utilizing the publicly available RNA-seq dataset published in Phytozome (Libault et al., 2010) (Table S2). As shown in Figure 5, the expression patterns of soybean trihelix genes are generally classified into 5 groups. Those genes in Group 1 are highly expressed in most of the tested tissues in soybean. The group 2 genes also display high expression signals but seems to preferentially express in some tissues, such as Glyma.10G161200 and Glyma.20G224000 which show markedly higher transcript abundance profiles in SAM, pods and seeds, but lower expression in roots, nodules and flowers. Group 3 contains 3 genes which are highly expressed in root hairs, nodules and seeds. Genes in Group 4 show low expression levels in almost all of the tissues. It is possible that expression of these genes is induced by a particular condition or that they are pseudogenes. Group 5 contains 30 trihelix genes, of which all of them display relatively low expression levels in most of the tissues, but higher in particular tissues, such as Glyma.04G135400, Glyma.11G156700, Glyma.10G202300, Glyma.03G191700 and Glyma.19G192300, which exhibit low transcript abundance in all of the tested tissues but with high expression levels in stems (Figure 5). 


\section{Cis-element analysis of soybean trihelix genes}

273 In order to predict the functions of soybean trihelix genes, the cis-elements in the promoter

274 regions were analyzed. As shown in Figure S4, 94.37\% of the soybean trihelix genes possess at 275 least one cis-element involved in light responsiveness, which is consistent with the light276 responsive characteristic of trihelix TFs (Green et al., 1987; Zhou et al., 1999). Meanwhile, 3

277 cis-elements related to abiotic stresses were identified, including the anaerobic responsive 278 element (ARE) (39.44\%), drought-responsive element (DRE and MBS) (19.72\%) and low 279 temperature responsive element (LTR) (7.04\%). Notably, ARE is much more widely distributed 280 than the other 2 cis-elements related to abiotic stresses (existed in 28 out of $71(39.4 \%)$ trihelix 281 genes) (Figure S4, Figure 6). Hormone response elements such as abscisic acid responsive 282 element (ABRE) (43.66\%), methyl jasmonate responsive elements (TGACG-motif) (47.89\%), 283 ethylene-responsive elements (ERE) (59.15\%) and salicylic acid responsive elements (TCA284 element) (14.08\%) were also analyzed and displayed in Figure 6 and Table S3. All of the 71 285 trihelix genes (except Glyma.01G150500) possess at least 1 stress-responsive cis-element 286 (Figure 6). These results suggest that trihelix genes may be involved in responses to abiotic and 287 biotic stresses.

\section{8}

289

290

291

292

293

294

295

296

297 298

299

\section{Mitogen-activated protein kinase (MAPK)-specific phosphorylation sites and miRNA target prediction}

It was reported that MAMP (microbe-associated molecular patterns) treatments induced rapid phosphorylation of an Arabidopsis trihelix gene, ASR3, via MAP KINASE4 (Li et al., 2015).

This fact suggests that trihelix genes could be post-translationally regulated by phosphorylation. Therefore, we also predicted the presence of putative MAPK phosphorylation sites. 29 soybean trihelix genes which are distributed in all of the 6 subfamilies have at least one putative MAPK phosphorylation site. Among them, 22 (75.9\%) belong to the GT-2 subfamily (containing 9 trihelix genes have at least one MAPK phosphorylation site) and SIP1 subfamily (containing 13 trihelix genes have at least one MAPK phosphorylation site). In the GT-2 subfamily, the putative MAPK phosphorylation sites are mostly threonine residue. While in the SIP1 subfamily, the putative MAPK phosphorylation sites are mostly serine residues (Table S4).

MicroRNAs (miRNAs) are a class of small non-coding regulatory RNAs that regulate gene expression by guiding target mRNA cleavage or translational inhibition (Unver et al., 2009; 
302 Eldem et al. 2013; Zhang., 2015). In order to identify the involvement of miRNAs in regulating

303 the expression of soybean trihelix genes, putative miRNA targets were also analyzed. As shown

304 in Table S5, 26 soybean trihelix genes were predicted to be targeted by 27 miRNAs, with

305 inhibition of cleavage or translation. Glyma.11G247300, Glyma.19G243500 and

306 Glyma.17G128500 contain three target sites; Glyma.18G010000, Glyma.07G151100,

307 Glyma.02G082100 and Glyma.10G225300 contain two target sites; the other 19 trihelix genes

308 have only one target site. Additionally, both of the gma-miR1533 and gma-miR1512a-3p can

309 target 3 of the trihelix genes.

310 The expression profile of soybean trihelix genes in response to environmental stresses

311 Previous studies showed that the maize trihelix genes could respond to flood stress ( Du et al.,

312 2016). Considering that many soybean trihelix genes processed the anaerobic responsive element

313 (ARE) in their promoters, we analyzed our previous transcriptome data of the soybean cultivar

314 Qihuang34 submerged for 3, 6, 12 and 24 hours at the seedling stage (Lin et al., 2019) (Table S6).

31545 trihelix genes display more than 2-fold expression level differences compared with controls in

316 at least one time point (Figure 7, Table S7). 25, 25, 37 and 31 differentially expressed genes

317 (DEGs) were identified after submergence treatment at 3, 6, 12 and 24 h, respectively (Table S7).

31815 soybean trihelix genes are significantly changed at all of the 4 time points (Table 2 ). These 15

319 DEGs are distributed in 5 subfamilies including SIP1, SH4, GT $\gamma$, GT-1 and GT-2 (Table 2).

320 Among these 15 DEGs, 4 genes (Glyma.11G156700, Glyma.20G224000, Glyma.05G200400,

321 and Glyma.08G007800) are down-regulated, and the remaining genes are all up-regulated. We

322 then performed qRT-PCR assays of these 15 DEGs with the root samples from the Qihuang 34

323 plants suffered submergence (Figure S5). As shown in Figure S5, the expression of 11 genes

324 (Glyma03G189600, Glyma06G149900, Glyma07G042800, Glyma07G151100,

325 Glyma08G007800, Glyma09G116400, Glyma11G247300, Glyma.13G149700,

326 Glyma13G229600, Glyma15G082900 and Glyma19G19000) is significantly changed under any

327 timepoint, which is consistent with our previous RNA-Seq results. Notably, 3 GT $\gamma$ subfamily

328 genes (Glyma.03G189600, Glyma.13G149700 and Glyma.19G190000) are all up-regulated, and

329 represent the most significant change (Table 2, Figure S5). The other 4 genes (Glyma11G157600,

330 Glyma16G167600, Glyma20G224000 and Glyma05G200400) are only up- or down-regulated at

331 specific time points after flood treatment (Figure S5).

Peer] reviewing PDF | (2019:08:40490:3:0:NEW 29 Jan 2020) 
332 To gain more insights into the role of trihelix genes in other abiotic stress responses, we 333 performed qRT-PCR assays to examine the expression profiles of 12 selected trihelix genes 334 under high-salt stress and ABA treatment (Figure 8). These 12 genes includes the 3 GT $\gamma$ 335 subfamily genes (Glyma.03G189600, Glyma.13G149700 and Glyma.19G190000) which 336 represent the most significant change under flood treatment and other 9 randomly selected 337 trihelix genes. According to the results, the expression of 6 genes is changed when plants were 338 treated with $\mathrm{NaCl}$ (Figure 8 A-L). 3 genes (Glyma.09G1 16400, Glyma.13G195800 and 339 Glyma20G166800) are down-regulated by $\mathrm{NaCl}$ treatment, while 3 genes (Glyma.03G189600, 340 Glyma.13G149700 and Glyma19G190000) are up-regulated $6 \mathrm{~h}$ after $\mathrm{NaCl}$ treatment. The other 3416 genes didn't show obvious changes (Figure 8A-L). As for the ABA treatment (Figure 8M-X), 1 342 trihelix gene (Glyma.07G151100) didn't show obvious change compared to the control, while 343 the other 11 tested trihelix genes were up- or down-regulated under ABA treatment (Figure 8M$344 \mathrm{X}$ ). Among them, Glyma.13G195800 is down regulated $3 \mathrm{~h}$ after ABA treatment, and the other 34510 genes are up-regulated at different time point (Figure 8M-X). It is noteworthy that the $3 \mathrm{GT} \gamma$ 346 genes (Glyma.03G189600, Glyma.13G149700 and Glyma19G190000) which are extremely sensitive to flooding are all significantly up-regulated by $\mathrm{NaCl}$ treatment and $\mathrm{ABA}$ treatment at

348 349 350

351

352

353

354

355 356

357

358 359 360 an exact time point (Table 2, Figure 8).

It has been shown that trihelix genes may be involved in plant defense responses. Microbeassociated molecular pattern (MAMP)-triggered immunity (MTI) is an important component of the plant innate immunity response to invading pathogens (Kim et al., 2011). In the current study, we used the publicly available microarray data (series accession no. GSE32642) to analyze the expression of trihelix genes in soybean leaves which were treated by the MAMP mixture (flg22 and chitin) (Valdés-López et al., 2011). This analysis showed the expression of 2 soybean trihelix genes were significantly changed $30 \mathrm{~min}$ after the MAMP mixture treatment (Figure 9A, Table S8). Glyma.01G121000 is significantly up-regulated, while Glyma.10G066800 is downregulated. Phytohormones, such as JA, SA and ethylene regulate plant defenses against diverse pathogens. We next examined the expression pattern of these 2 trihelix genes in soybean leaves under JA, SA and ACC treatment by qRT-PCR. As shown in figure 9, both of these two genes are sensitive to SA, JA and ACC. Glyma.01G121000 is up-regulated under all of these treatments, while Glyma.10G066800 displays a more complex expression pattern. Under SA treatment, Glyma.10G066800 is up-regulated $30 \mathrm{~min}$ and 12 hours after treatment, but is down- 
363 regulated 3 hours after treatment. Under JA treatment, Glyma.10G066800 is up-regulated 30

364 minutes after treatment, but is down-regulated 3 and 12 hours after treatment. Under ACC

365 treatment, it is down regulated at $3 \mathrm{~h}$ and $12 \mathrm{~h}$ after treatment.

366

\section{Discussion}

368 Characterization of the soybean trihelix genes

369

370

371

372

373

374

375

376

377

378

379

380

381

382

383

384

385

386

387

388

389

390

391

392

393

In this study, we identified 71 soybean trihelix genes by the Myb/SANT-LIKE domain using an HMM (Hidden Markow Model)-search. We obtained more trihelix genes in the present study than in the previous report (Osorio et al., 2012) because the trihelix genes were identified in the new version of the soybean genome database Wm82.a2. v1 and performing a different search method. To date, the soybean trihelix family is the second largest known trihelix gene family (next to the wheat trihelix family composed of 94 trihelix genes) (Xiao et al., 2019) compared with other plants, such as Arabidopsis (28), rice (41) tomato (36), Brassica rapa (52), Chrysanthemum (20) and tartary buckwheat (31) (Yasmeen et al., 2016; Yu et al., 2015; Song et al., 2016; Wang et al., 2017; Wang et al., 2018; Li et al., 2019; Ma et al., 2019).

Gene duplication is one of the major evolutionary mechanisms for generating novel genes that help organisms adapt to different environments (Moore et al., 2003; Kong et al., 2007). In general, gene families expand mainly by tandem and segmental duplications (Kong et al., 2007). Only 13 pairs of segmental duplicated genes (36.6\%) were identified in soybean among total 71 trihelix genes (Figure 2, Table 1). This finding suggests that more than half of the trihelix genes in soybean may not originate from the same ancestor. A similar phenomenon was reported in the rice trihelix gene family: it contained only six pairs of duplicated genes among a total of 41 (29.3\%) rice trihelix genes (Li et al., 2019). The Soybean genome has undergone two rounds of whole genome duplication events at approximately 59 and 13 million years ago (Schmutz et al., 2010). In the present study, all of the segmental duplication events in the soybean gene trihelix family occurred during the recent whole genome duplication event when glycine-specific duplication occurred (Table 1). The duplicated genes which experience subfunctionalization through purifying selective pressure $(\mathrm{Ka} / \mathrm{Ks}<1)$ (Cusack et al., 2007), neofunctionalization through positive selective pressure (Ka/Ks > 1) (Blanc et al., 2004) and nonfunctionalization play important roles in adaptive evolution (Flagel et al., 2009). In this study, all of the $\mathrm{Ka} / \mathrm{Ks}$ ratios in different trihelix gene pairs are less than 1 (Table 1), suggesting that all of those 
394 duplicated genes experience a strong purifying selective pressure and subfunctionalized during 395 evolution. This phenomenon is consistent with the fact that most duplicated genes are 396 subfunctionalized in soybean (Roulin et al., 2013).

397 Trihelix family genes had been classified into three distinctive subfamilies (GT $\alpha$, GT $\beta$, 398 and GT $\gamma$ ) previously (Fang et al., 2010). Then, Kaplan-Levy et al (2012) classified trihelix genes 399 from rice (Oryza sativa) and Arabidopsis into five clades, named GT-1, GT-2, SH4, SIP1, and 400 GT $\gamma$. Recently, a new subfamily, GT $\delta$ was formed in tomato (Solanum lycopersicum) and rice 401 (Yu et al., 2015; Li et al., 2019). In the present study, the classification of the soybean trihelix 402 gene family has been renewed according to their new classification in Arabidopsis, tomato and 403 rice (Figure 3) (Kaplan et al., 2012; Yasmeen et al., 2016; Yu et al., 2015; Li et al., 2019). All of 404 the 71 soybean trihelix genes were classified into 6 subfamilies (GT-1, GT-2, SH4, SIP1, GT $\gamma$ 405 and GT $\delta$ ). The 3 genes in the GT $\delta$ subfamily have a large number of exons (17 exons), depicting 406 a significant difference in gene structure from the other subfamily genes (Figure 4). This finding 407 is similar to SIGT-12, a member of the GT $\delta$ subfamily in tomato (Yu et al., 2015). In addition, 40868 soybean trihelix genes were predicted to localize to the nucleus, and only 3 were predicted to 409 localize to the chloroplast (Table S1). We noticed that two of those chloroplast localized genes 410 were from the GT $\delta$-subfamily. Therefore, the members in the GT $\delta$ subfamily might serve very 411 different functions from trihelix genes in other subfamilies. Although most of the trihelix genes 412 in soybean may not originate from the same ancestor, the type and number of motifs of soybean 413 trihelix proteins were generally similar within the same sub-family (Figure 4). Furthermore, 414 members in the same sub-family generally share similar structures and exon numbers (Figure 4). 415 These results suggest that the trihelix genes belonging to the same subfamily might play similar 416 roles in soybean development, and the conserved motifs may play roles in subfamily-specific 417 functions.

\section{Expression analysis of soybean trihelix genes}

Expression analyses could provide insight into the potential functions of genes. Therefore, 422 the expression pattern of 71 trihelix genes in different tissues of soybean was performed using publicly available RNA-seq data. Many tested trihelix genes were broadly expressed, while there was also a high number of trihelix genes exhibiting specific expression patterns (Figure 5). Among the 13 pairs of duplicate genes, more than half of the duplicate gene pairs showed similar 
425 expression between the duplicates (Figure S6). For example, Gm04G216100 and its paralog 426 Glyma.09G116400 had higher expression in leaves. Gm13G195800 and its paralog 427 Glyma15G234100 had higher expression in nodules, stem and pod, suggesting that they might 428 have redundant roles. 5 duplicated genes pairs seemed to have different expression patterns. For 429 example, Gm09G116400 was highly expressed in leaves, while its paralog Glyma.06G149900 430 had higher expression in root and flower. Gm04G194600 had a relative higher expression level 431 in flower, while its paralog Gm06G171400 had higher expression in SAM and leaves. This 432 finding suggests that they might have different functions.

433 Nowadays, various areas in the world face abiotic stresses, such as flooding and salinity. 434 The involvement of the stress responsive genes in various metabolic processes contributes to 435 enhancing stress tolerance in plants. It has been reported that trihelix genes could respond to the 436 waterlogging and salt stress (Fang et al., 2010; Xie et al., 2009; Xi et al., 2012; Giuntoli et al., 2014; Luo et al., 2017; Du et al., 2016; Magwanga et al., 2019). Lack of oxygen has been proposed as the main problem associated with flooding (Drew., 1983; Drew., 1997). In this study, $39.4 \%$ of the soybean trihelix genes contain at least one anaerobic responsive element (ARE) in their promoters (Figure 6, Table S3), implying that the soybean trihelix genes might be

441

442 443

444 445

446

447 448 449 450 451 452 453 454 455 involved in flood response. 11 trihelix genes that respond to flood stress at all of the 4 time points were identified in this study (Figure 7, Table 2, Figure S5), and all of these genes contained at least 1 stress-responsive cis-element (Figure 6). Among them, 3 genes (Glyma.03G189600, Glyma.13G149700 and Glyma.19G190000) which belong to the GTy subfamily showed the highest sensitivity to the submergence treatment (Table 2). Group VII ethylene response factors (ERF-VIIs) are a class of ERF TFs that regulate the expression of a wide range of genes involved in adaptive responses to flooding and low oxygen levels (Voesenek and Bailey-Serres., 2015). It has been reported that HRA1 (At3G10040), a submergence or hypoxia-inducible GT $\gamma$ trihelix gene in Arabidopsis, acted as a negative regulator of an ERF-VII TF RAP2.12, and then reducing the expression of core hypoxia-response genes (Giuntoli et al., 2014). This regulatory mechanism may contribute to the avoidance of RAP2.12 overaccumulation, preventing rapid depletion of carbohydrate reserves under oxygen deprivation (such as during submergence) (Fukao et al., 2019). In the present study, of the 3 GT $\gamma$ genes mentioned above, the expression level of Glyma.19G190000, which is homologous to HRAl was also strongly induced by submergence treatment, suggesting its potential role in flood tolerance. 
456 Until now, none of the flood-responsive trihelix genes have been characterized in soybean. The 457 trihelix genes which were continuously induced by submergence treatment, specifically 458 Glyma.19G190000 could be candidate genes for further study on flood tolerance in soybean.

459 In addition, our study also found that certain trihelix genes could respond to high-salt stress 460 (Figure 8A-L). Previously, a soybean trihelix gene, GmGT-2A (Glyma.04G216100), was 461 reported to be up-regulated by $\mathrm{NaCl}$. Moreover, overexpression of the $G m G T$ - $2 A$ improved plant 462 tolerance to salt (Xie et al., 2009). In our study, no significant change in its expression level was 463 detected under $\mathrm{NaCl}$ treatment (Figure 8B). This different expression pattern might result from 464 the different external $\mathrm{NaCl}$ concentration (Xie et al used $150 \mathrm{mM} \mathrm{NaCl}$ but we used $100 \mathrm{mM}$ $465 \mathrm{NaCl}$ in this study). Perhaps the lower $\mathrm{NaCl}$ concentration used in this study was not high 466 enough to induce its expression. In rice, 3 trihelix genes belonging to the GT $\gamma$ subfamily (OsGT$467 \gamma-1, O s G T \gamma-2$ and $O s G T \gamma-3)$ were strongly induced by abiotic stress such as drought, cold and 468 salt. In addition, overexpression of $O s G T \gamma-1$ in rice enhanced salt tolerance at the seedling stage, 469 suggesting that the OsGT $\gamma$ subfamily may participate in the regulation of stress tolerance in rice 470 (Fang et al., 2010). In our study, we found that the 3 GT $\gamma$ genes (Glyma.03G189600, 471 Glyma.13G149700 and Glyma.19G190000) which were showing the highest sensitivity to 472 submergence were also induced by $\mathrm{NaCl}$. This finding suggests that the GT $\gamma$ subfamily genes in 473 soybean may have potential functions in the stress tolerance as in rice.

474 Abscisic acid (ABA), which is commonly referred to a stress hormone, is involved not only 475 in regulating stomatal opening, growth and development but also in abiotic stress responses in 476 plants (Tuteja, 2007). Some trihelix genes were found to respond to ABA treatment (Xie et al., 477 2009; Fang et al., 2010; Xi et al., 2012). Notably, the BnSIP1-1 gene from Brassica napus was 478 reported to play roles in ABA synthesis and signaling, salt and osmotic stress response (Luo et $479 a l ., 2017)$. In this study, among the 71 trihelix genes in soybean, 31 genes contain ABA480 responsive elements (Figure 6, Tables S3). By performing qRT-PCR, all of the selected genes 481 (except Glyma.07G151100) were found to indeed be regulated by ABA treatment. We focused 482 on the expression of the 3 GT $\gamma$ genes mentioned above and found that all of them could respond 483 to ABA treatment at a specific time point. This result was in concordance with the fact that all of 484 them possess ABA-responsive elements (Figure 6, Table S3). Taken together, the 3 GT $\gamma$ genes 485 of the trihelix family could be the best candidates for further study of abiotic stress tolerance in 486 soybean. 
487

488

489

490

491

492

493

494

495

496

497

498

499

500

501

502

503

504

505

506

507

508

509

510

511

512

513 References

514 Ahmed F, Rafii MY, Ismail MR, Juraimi AS, Rahim HA, Asfaliza R, Latif MA (2012)

515 Waterlogging tolerance of crops: breeding, mechanism of tolerance, molecular approaches, and

516 future prospects. Biomed Res Int 2013: 963525.

component of the plant innate immunity and responses to invading pathogens (Kim et al., 2011). In Arabidopsis, trihelix TF GT2-like 1 (GTL1) plays an important role in coordinating plant immunity (Völz et al., 2018). Moreover, ASR3 functions as a transcriptional repressor regulated by MAMP-activated MPK4 to fine-tune plant immune gene expression ( $L i$ et al., 2015). In the present study, Glyma.01G121000 was induced by MAMP mixture, JA, SA and ACC treatments, suggesting that it might also be involved in coordinating plant immunity in soybean. This initial research of soybean trihelix genes should be followed by further work that focuses on plant transformation and phenotyping in order to thoroughly explore trihelix functions in abiotic and biotic stress responses, and the crosstalk between this gene family and hormone signals.

\section{Conclusions}

In conclusion, 71 members of the trihelix gene family were identified in the newest version of the soybean genome and were classified into 6 subfamilies based on phylogenetic relationships. Genes in the same subfamilies generally share similar gene structure and conserved functional domains. 13 segmental duplicated gene pairs were identified and all of them experienced a strong purifying selective pressure during evolution. By investigating the differential expression profiles of the trihelix genes under flooding, high-salt stresses and MAMP treatment, several candidates for further study of soybean stress-tolerance were identified. These results provide a basis to elucidate the function and the molecular mechanism of trihelix genes on plant development and stress tolerance.

Acknowledgements

We thank Dr. Zhanji Liu for his assistance on bioinformatics analysis during the manuscript preparation. 
517 Artimo P, Jonnalagedda M, Arnold K, Baratin D, Csardi G, Castro E, Duvaud S, Flegel V, 518 Fortier A, Gasteiger E, Grosdidier A, Hernandez C, Ioannidis V, Kuznetsov D, Liechti R, 519 Moretti S, Mostaguir K, Redaschi N, Rossier G, Xenarios I, Stockinger H (2012) ExPASy: SIB 520 bioinformatics resource portal. Nucleic Acids Res 40: W597-W603.

521 Bailey TL, Johnson J, Grant CE, Noble WS (2015) The MEME suite. Nucleic Acids Res 43: 522 W39-W49.

523 Barr MS, Willmann MR, Jenik PD (2012) Is there a role for trihelix transcription factors in 524 embryo maturation? Plant Signal Behav 7: 205-209.

525 Belamkar V, Weeks NT, Bharti AK, Farmer AD, Graham MA, Cannon SB (2014)

526 Comprehensive characterization and RNA-Seq profiling of the HD-Zip transcription factor 527 family in soybean (Glycine max) during dehydration and salt stress. BMC genomics15: 950. 528 Blanc G, Wolfe KH (2004) Functional divergence of duplicated genes formed by polyploidy 529 during Arabidopsis evolution. The Plant Cell 16: 1679-1691.

530 Breuer C, Kawamura A, Ichikawa T, Tominaga-Wada R, Wada T, Kondou Y, Muto S, Matsui 531 M, Sugimoto K (2009) The trihelix transcription factor GTL1 regulates ploidy-dependent cell 532 growth in the Arabidopsis trichome. The Plant Cell 21: 2307-2322.

533 Chen W, Yao Q, Patil GB, Agarwal G, Deshmukh RK, Lin L, Wang B, Wang Y, Prince SJ, 534 Song L, Xu D, An YC, Valliyodan B, Varshney, RK, Nguyen HT (2016) Identification and 535 comparative analysis of differential gene expression in soybean leaf tissue under drought and 536 flooding stress revealed by RNA-Seq. Front Plant Sci 7: 1044.

537 Cusack BP, Wolfe KH (2007) When gene marriages don't work out: divorce by 538 subfunctionalization. Trends in genetics 23: 270-272.

539 Dai X, Zhuang Z, Zhao PX (2018) psRNATarget: a plant small RNA target analysis server (2017 540 release). Nucleic Acids Research 46: W49-W54.

541 Drew M (1983) Plant injury and adaptation to oxygen deficiency in the root environment: A 542 review. Plant Soil 75: 179-199.

543 Drew MC (1997) Oxygen deficiency and root metabolism: Injury and acclimation under hypoxia 544 and anoxia. Annu Rev Plant Biol 48: 223-250.

545 Du H, Huang M, Liu L (2016) The genome wide analysis of GT transcription factors that 546 respond to drought and waterlogging stresses in maize. Euphytica 208: 113-122. 
547 Eldem V, Okay S, Unver T (2013) Plant microRNAs: new players in functional genomics. Turk 548 J Agric For 37:1-21.

549 Fang Y, Xie K, Hou X, Hu H, Xiong L (2010) Systematic analysis of GT factor family of rice

550 reveals a novel subfamily involved in stress responses. Mol Genet Genomics 283: 157-169.

551 Flagel LE,Wendel JF (2009) Gene duplication and evolutionary novelty in plants. New 552 Phytologist 183: 557-564.

553 Finn RD, Coggill P, Eberhardt RY, Eddy SR, Mistry J, Mitchell AL, Potter SC, Punta M,

554 Qureshi M, Sangrador-Vegas A, Salazar GA, Tate J, Salazar GA (2015) The Pfam protein

555 families database: towards a more sustainable future. Nucleic Acids Res44: D279-D285.

556 Fukao T, Barrera-Figueroa BE, Juntawong P, Peña-Castro JM (2019) Submergence and

557 Waterlogging Stress in Plants: A Review Highlighting Research Opportunities and Understudied 558 Aspects. Frontiers in Plant Science10:340.

559 Gao MJ, Lydiate DJ, Li X, Lui H, Gjetvaj B, Hegedus DD, Rozwadowski K (2009) Repression

560 of seed maturation genes by a trihelix transcriptional repressor in Arabidopsis seedlings. The

561 Plant Cell 21: 54-71.

562 Gao J, Thelen JJ, Dunker AK, Xu D (2010) Musite, a tool for global

563 prediction of general and kinase-specific phosphorylation sites. Mol Cell Proteomics 9: 2586-

5642600.

565 Giuntoli B, Lee SC, Licausi F, Kosmacz M, Oosumi T, van Dongen JT, Serres JB, Perata, P

566 (2014). A trihelix DNA binding protein counterbalances hypoxia-responsive transcriptional

567 activation in Arabidopsis. Plos Biol 12: e1001950.

568 Goodstein DM, Shu S, Howson R, Neupane R, Hayes RD, Fazo J, Mitros T, Dirks W, Hellsten

569 U, Putnam N, Rokhsar DS (2011) Phytozome: a comparative platform for green plant genomics.

570 Nucleic Acids Res 40: D1178-D1186.

571 Green PJ, Kay SA, Chua NH (1987) Sequence-specific interactions of a pea nuclear factor with

572 light-responsive elements upstream of the $r b c S-3 A$ gene. EMBO J 6: 2543-2549.

$573 \mathrm{Hu}$ B, Jin J, Guo AY, Zhang H, Luo J, Gao G (2014) GSDS 2.0: an upgraded gene feature

574 visualization server. Bioinformatics 31: 1296-1297.

575 Kaplan-Levy RN, Brewer PB, Quon T, Smyth DR (2012) The trihelix family of transcription

576 factors-light, stress and development. Trends Plant Sci 17: 163-171.

577 Kim M, Hyten DL, Niblack TL, Diers BW (2011) Stacking resistance alleles from 
578 wild and domestic soybean sources improves soybean cyst nematode resistance. Crop

579 Sci 51: 934-943.

580 Koberg M, Abu-Much R, Gedanken A (2011) Optimization of bio-diesel production from

581 soybean and wastes of cooked oil: Combining dielectric microwave irradiation and a $\mathrm{SrO}$

582 catalyst. Bioresource Technol 102: 1073-1078.

583 Kong H, Landherr LL, Frohlich MW, Leebens-Mack J, Ma H, DePamphilis CW (2007) Patterns

584 of gene duplication in the plant SKP1 gene family in angiosperms: evidence for multiple

585 mechanisms of rapid gene birth. Plant J 50: 873-885.

586 Kumar S, Stecher G, Tamura K (2016) MEGA7: molecular evolutionary genetics analysis

587 version 7.0 for bigger datasets. Mol Biol Evol 33: 1870-1874.

588 Libault M, Farmer A, Joshi T, Takahashi K, Langley RJ, Franklin LD, He J, Xu D, May G, 589 Stacey G (2010) An integrated transcriptome atlas of the crop model Glycine max, and its use in 590 comparative analyses in plants. Plant J 63: 86-99.

591 Li B, Jiang S, Yu X, Cheng C, Chen S, Cheng Y, Yuan J, Jiang D, He P, Shan, L (2015)

592 Phosphorylation of trihelix transcriptional repressor ASR3 by MAP KINASE4 negatively 593 regulates Arabidopsis immunity. The Plant Cell 27: 839-856.

594 Li S, Wang N, Ji D, Xue Z, Yu Y, Jiang Y, Liu J, Liu Z, Xiang F (2016) Evolutionary and 595 functional analysis of membrane-bound NAC transcription factor genes in soybean. Plant 596 physiology 172: 1804-1820.

597 Li J, Zhang M, Sun J, Mao X, Wang J, Wang J, Liu H, Zheng H, Zhen Z, Zhao H, Zou D (2019)

598 Genome-Wide Characterization and Identification of Trihelix Transcription Factor and

599 Expression Profiling in Response to Abiotic Stresses in Rice (Oryza sativa L.). Int J Mol Sci 20: 600251.

601 Lin Y, Li W, Zhang Y, Xia C, Liu Y, Wang C, Xu R, Zhang L (2019) Identification of 602 genes/proteins related to submergence tolerance by transcriptome and proteome analyses in 603 soybean. Sci Rep-UK 9: 14688.

604 Livak KJ, Schmittgen TD (2001) Analysis of relative gene expression data using real-time 605 quantitative PCR and the $2^{-\Delta \Delta C T}$ method. Methods 25: 402-408.

606 Luo J, Tang S, Mei F, Peng X, Li J, Li X, Yan X, Zeng X, Liu F, Wu Y, Wu G (2017) BnSIP1-1, 607 a trihelix family gene, mediates abiotic stress tolerance and ABA signaling in Brassica napus. 608 Front Plant Sci 8: 44. 
609 Lynch M, Conery JS (2000) The evolutionary fate and consequences of duplicate genes. Science 610 290: 1151-1155.

611 Magwanga RO, Kirungu JN, Lu P, Yang X, Dong Q, Cai X, Xu Y, Wang X, Zhou Z, Hou Y,

612 Nyunja R, Agog S, Hua J, Zhang B, Wang K, Liu F (2019) Genome wide identification of the

613 Trihelix transcription factors and overexpression of Gh_A05G2067 (GT-2), a novel gene

614 contributing to increased drought and salt stresses tolerance in cotton. Physiol Plantarum 167:

$615 \quad 447-464$.

616 Ma Z, Liu M, Sun W, Huang L, Wu Q, Bu T, Li C, Chen, H (2019) Genome-wide identification 617 and expression analysis of the trihelix transcription factor family in tartary buckwheat

618 (Fagopyrum tataricum). BMC Plant Biol 19: 344.

619 Moore RC, Purugganan MD (2003) The early stages of duplicate gene evolution. P Natl Acad 620 Sci USA 100: 15682-15687.

621 Nanjo Y, Maruyama K, Yasue H, Yamaguchi-Shinozaki K, Shinozaki K, Komatsu S (2011)

622 Transcriptional responses to flooding stress in roots including hypocotyl of soybean seedlings.

623 Plant Mol Biol 77: 129-144.

624 Nei M, Sudhir K (2000) Molecular evolution and phylogenetics. Oxford university: New York, 625 USA.

626 Osorio MB, Bücker-Neto L, Castilhos G, Turchetto-Zolet AC, Wiebke-Strohm B, Bodanese-

627 Zanettini MH, Margis-Pinheiro M (2012) Identification and in silico characterization of soybean

628 trihelix-GT and bHLH transcription factors involved in stress responses. Genet Mol Biol 35:

$629 \quad 233-246$.

630 Qin Y, Ma X, Yu G, Wang Q, Wang L, Kong L, Kim W, Wang H (2014) Evolutionary history of 631 trihelix family and their functional diversification. DNA Res 21: 499-510.

632 Roulin A, Auer PL, Libault M, Schlueter J, Farmer A, May G, Stacey G, Doerge RW, Jackson, 633 SA (2013) The fate of duplicated genes in a polyploid plant genome. The Plant Journal 73: 143634153.

635 Schmutz J, Cannon SB, Schlueter J, Ma J, Mitros T, Nelson W, Hyten D, Song Q, Thelen JJ, 636 Cheng J, Xu D, Hellsten U, May GD, Yu Y, Sakura T, Umezawa T, Bhattacharyya MK, Sandhu 637 D, Valliyodan B, Lindquist E, Peto M, Grant D, Shu S, Goodstein D, Barry K, Futrell-Griggs M, 638 Abernathy B, Du J, Tian Z, Zhu L, Gil IN, Joshi T, Libault M, Sethuraman A, Zhang X, 639 Shinozaki K, Nguyen HT, Wing RA, Cregan P, Specht J, Grimwood J, Rokhsar D, Stacey G, 
640 Shoemaker RC, Jackson SA (2010) Genome sequence of the palaeopolyploid soybean. Nature 641 463: 178.

642 Song A, Wu D, Fan Q, Tian C, Chen S, Guan Z, Xin J, Zhao K, Chen F (2016) Transcriptome-

643 wide identification and expression profiling analysis of chrysanthemum trihelix transcription

644 factors. Int J Mol Sci 17: 198.

645 Song L, Valliyodan B, Prince S, Wan J, Nguyen H (2018) Characterization of the XTH Gene

646 Family: New Insight to the Roles in Soybean Flooding Tolerance. Int J Mol Sci 19: 2705.

647 Tuteja N (2007) Abscisic acid and abiotic stress signaling. Plant signaling \& behavior 2: 135648138.

649 Tyler BM (2007) Phytophthora sojae: root rot pathogen of soybean and model oomycete. Mol 650 Plant Pathol 2007:1-8

651 Tzafrir I, Pena-Muralla R, Dickerman A, Berg M, Rogers R, Hutchens S, Sweeney TC, McElver

652 J, Aux G, Patton D, Meinke D (2004) Identification of genes required for embryo development

653 in Arabidopsis. Plant Physiol 135: 1206-1220.

654 Unver T, Namuth-Covert DM, Budak H (2009) Review of current methodological approaches

655 for characterizing microRNAs in plants. Int J Plant Genomics 2009: 262463

656 Valdés-López O, Thibivilliers S, Qiu J, Xu WW, Nguyen TH, Libault M, Le BH, Goldberg RB,

657 Hill CB, Hartman GL, Diers B, Stacey G (2011) Identification of quantitative trait loci controlling

658 gene expression during the innate immunity response of soybean. Plant physiol 157: 19751986.

659 Voorrips RE (2002) MapChart: software for the graphical presentation of linkage maps and 660 QTLs. J Hered 93: 77-78.

661 Voesenek LA, Bailey-Serres J (2015) Flood adaptive traits and processes: an overview. New 662 Phytol 206: 57-73.

663 Völz R, Kim SK, Mi J, Mariappan KG, Guo X, Bigeard J, Alejandro S, Pflieger D, Rayapuram

664 N, Al-Babili S, Hirt H (2018) The Trihelix transcription factor GT2-like 1 (GTL1) promotes

665 salicylic acid metabolism, and regulates bacterial-triggered immunity. PLoS genetics 14:

666 e1007708.

667 Wang D, Shannon MC (1999) Emergence and seedling growth of soybean cultivars and maturity 668 groups under salinity. Plant and soil 214: 117-124. 
669 Wang W, Wu P, Liu T, Ren H, Li Y, Hou X (2017) Genome-wide Analysis and Expression

670 Divergence of the Trihelix family in Brassica rapa: Insight into the Evolutionary Patterns in 671 Plants. Sci Rep-UK 7: 6463.

672 Wang Z, Zhao K, Pan Y, Wang J, Song X, Ge W, Yuan M, Lei T, Wang L, Zhang L, Li Y, Liu

673 T, Chen W, Meng W, Sun C, Cui X, Bai Y, Wang X (2018) Genomic, expressional, protein-

674 protein interactional analysis of Trihelix transcription factor genes in Setaria italia and inference

675 of their evolutionary trajectory. BMC Genomics 19: 665.

676 Wang Y, Jiang Z, Li Z, Zhao Y, Tan W, Liu Z, Cui S, Yu X, Ma J, Wang G, Li W (2019)

677 Genome-wide identification and expression analysis of the VQ gene family in soybean (Glycine 678 max). Peer J 7: e7509.

679 Wilcox JR (2004) World distribution and trade of soybean. In Soybeans: Improvement,

680 Production, and Uses. In: Boerma HG, Specht JE (Eds). Agronomy Monographs, 3rd edn.

681 Madison, WI, USA, Volume 16, pp. 1-14

682 Xi J, Qiu Y, Du L, Poovaiah BW (2012) Plant-specific trihelix transcription factor AtGT2L

683 interacts with calcium/calmodulin and responds to cold and salt stresses. Plant Sci 185: 274-280.

684 Xiao J, Hu R, Gu T, Han J, Qiu D, Su P, Feng J, Chang J, Yang G, He G (2019) Genome-wide

685 identification and expression profiling of trihelix gene family under abiotic stresses in wheat.

686 BMC genomics 20: 287.

687 Xie Z, Zou H, Lei G, Wei W, Zhou Q, Niu C, Liao Y, Tian A, Ma B, Zhang W, Zhang J, Chen S 688 (2009) Soybean Trihelix transcription factors GmGT-2A and GmGT-2B improve plant tolerance 689 to abiotic stresses in transgenic Arabidopsis. PloS ONE 4: e6898.

690 Yasmeen E, Riaz M, Sultan S, Azeem F, Abbas A, Riaz K, Ali MA (2016) Genome-wide 691 analysis of trihelix transcription factor gene family in Arabidopsis thaliana. Pak J Agr Sci 53: $692439-448$.

693 Yim AKY, Wong JWH, Ku YS, Qin H, Chan TF, Lam HM (2015) Using RNA-Seq data to 694 evaluate reference genes suitable for gene expression studies in soybean. PloS one 10:136343. 695 Y in X, Hiraga S, Hajika M, Nishimura M, Komatsu S (2016) Transcriptomic analysis reveals the 696 flooding tolerant mechanism in flooding tolerant line and abscisic acid treated soybean. Plant 697 Mol Biol 93: 479-496. 
698 Yoo CY, Pence HE, Jin JB, Miura K, Gosney MJ, Hasegawa PM, Mickelbart MV (2010) The 699 Arabidopsis GTL1 transcription factor regulates water use efficiency and drought tolerance by 700 modulating stomatal density via transrepression of SDD1. The Plant Cell 22: 4128-4141.

701 Yu C, Chen Y, Lu C, Hwang J (2006) Prediction of protein subcellular localization. Proteins 64: 702 643-651.

703 Yu C, Cai X, Ye Z, Li H (2015) Genome-wide identification and expression profiling analysis of 704 trihelix gene family in tomato. Bio chem Bioph Res Co 468: 653-659.

705 Yu Y, Wang N, Hu R, Xiang F (2016) Genome-wide identification of soybean WRKY

706 transcription factors in response to salt stress. Springerplus 5: 920.

707 Zhang Z, Li J, Zhao XQ, Wang J, Wong GKS, Yu J (2006) KaKs_Calculator: calculating Ka and

708 Ks through model selection and model averaging. Genom Proteom Bioinf 4: 259-263.

709 Zhang B (2015) MicroRNA: a new target for improving plant tolerance to abiotic stress. J Exp

710 Bot 66:1749-1761.

711 Zhou DX (1999) Regulatory mechanism of plant gene transcription by GT-elements and GT-

712 factors. Trends Plant Sci 4: 210-214.

713

714

715 Legends:

716 Figure 1. Chromosomal locations of soybean trihelix genes. Gray bars represent chromosomes.

717 Chromosome numbers are shown at the tops of the bar. Trihelix genes are labeled at the right of

718 the chromosomes. Scale bar on the left denotes the chromosome lengths (Mb). Chr:

719 Chromosome. A-S: 20 chromosomes.

720 Figure 2. Circle plot of soybean chromosomes and the 26 trihelix family genes displayed as 721 segmental duplicated gene pairs. The thick green lines indicate duplicated trihelix gene pairs.

722 Scale bar marked on the chromosome indicating chromosome lengths (Mb). Chr: Chromosome.

723 Figure 3. Phylogenetic tree based on protein sequences of trihelix genes in soybean, Arabidopsis 724 thaliana, rice and tomato. Gm, Glycine max; AT, Arabidopsis thaliana; Os, Oryza sativa; Solyc, 725 Solanum lycopersicon. The Phylogenetic tree was constructed using the neighbor-joining (NJ) 726 method, with 1000 bootstrap replicates, using MEGA v.7.0.

727 Figure 4. The conserved motifs and gene structure of the soybean trihelix family. The genes in 6 728 sub-families were marked with different colors. (A) The conserved motifs of the soybean trihelix 
729 family. The boxes with different colors on the right denote 10 motifs. A detailed motif

730 introduction is shown in Figure S2. The scale bar at the bottom indicates the lengths of the 731 trihelix protein sequences. (B) Gene structures of the soybean trihelix family. CDS (coding 732 sequence), introns, and untranslated regions (UTRs) are marked by yellow boxes, black lines, 733 and green boxes, respectively. The sizes of exons and introns can be estimated using the scale at 734 the bottom.

735 Figure 5. Heatmap of the expression profiles of the soybean trihelix genes in different tissues.

736 The expression values (Fragments per kilobase for a million reads, FPKM) for each gene in 737 different tissues were $\log 2$ transformed before generating heat map. SAM: Shoot Apical 738 Meristem. The expression data was obtained from the public RNA-seq data published in 739 Phytozome v12.1 (Libault et al., 2010; Goodstein et al., 2011).

740 Figure 6. Predicted cis-elements in the promoter regions (1500 bp upstream from the 741 transcription initiation site) of the soybean trihelix genes. The scale bar at the bottom indicates 742 the length of promoter sequence. ABRE: abscisic acid responsive element; ARE: anaerobic 743 responsive element; DRE: drought responsive element; ERE: ethylene responsive element; LTR:

744 low temperature responsive element; MBS: drought responsive element; TCA-element: salicylic 745 acid responsive element; TGACG-motif: methyl jasomonate responsive element.

746 Figure 7. Heatmap of the differentially expressed trihelix genes of soybean suffering from 747 submergence treatment. The RNA-seq data was obtained from NCBI Sequence Read Archive 748 (SRA) with the accession number: SRP181976 (Lin et al., 2019). The expression values 749 (Fragments per kilobase for a million reads, FPKM) for each gene were log2 transformed before 750 generating the heatmap. ck-3h, ck-6h, ck-12h and ck-24h indicate the roots from plants untreated 751 by submergence stress; F-3h, F-6h, F-12h, F-24h denote the roots sampled at $3 \mathrm{~h}, 6 \mathrm{~h}, 12 \mathrm{~h}$ and $75224 \mathrm{~h}$ after submergence stress treatment, respectively.

753 Figure 8. The expression profiles of 12 selected trihelix genes under $\mathrm{NaCl}$ and $\mathrm{ABA}$ treatment 754 examined by qRT-PCR. A-L: The relative expression levels of 12 selected trihelix genes under $755 \mathrm{NaCl}$ treatment. $0 \mathrm{~h}, 3 \mathrm{~h}, 6 \mathrm{~h}$, and $12 \mathrm{~h}$ denoted the roots sampled $0 \mathrm{~h}, 3 \mathrm{~h}, 6 \mathrm{~h}$, and $12 \mathrm{~h}$ after 100 756 $\mathrm{mM} \mathrm{NaCl}$ treatment, respectively. The data represent the mean $\pm \mathrm{SD}$ of three biological

757 replicates. The statistical significance was determined using Student's $t$-tests $(* * \mathrm{p}<0.01$, $758 * \mathrm{p}<0.05, \mathrm{n}=3$ ); M-X: The relative expression levels of 12 selected trihelix genes under ABA 759 treatment. $0 \mathrm{~h}, 3 \mathrm{~h}, 6 \mathrm{~h}$, and $12 \mathrm{~h}$ denoted the roots sampled $0 \mathrm{~h}, 3 \mathrm{~h}, 6 \mathrm{~h}$, and $12 \mathrm{~h}$ after $100 \mu \mathrm{M}$ 
760 ABA treatment, respectively. The data represent the mean $\pm \mathrm{SD}$ of three biological replicates.

761 The statistical significance was determined using Student's $t$-tests (** $\mathrm{p}<0.01, * \mathrm{P}<0.05, \mathrm{n}=3$ ).

762 Figure 9. The DEGs (differentially expressed genes) of soybean trihelix genes under MAMP

763 (microbe-associated molecular patterns) mixtures treatment and their expression patterns under

764 SA, JA and ACC treatment. A: 2 DEGs (Glyma.01G121000 and Glyma.10G066800) in soybean

765 leaves under MAMP (mixture with flg22 and chintin) treatment for $30 \mathrm{~min}$. The microarray data

766 was obtained from GEO series accession no. GSE32642 (Valdés-López et al., 2011). B-C: The

767 expression pattern of Glyma.01G121000 and Glyma.10G066800 in soybean leaves under SA

768 treatment examined by qRT-PCR. D-E: The expression pattern of Glyma.01G121000 and

769 Glyma.10G066800 in soybean leaves under JA treatment examined by qRT-PCR; F-G: The

770 expression pattern of Glyma.01G121000 and Glyma.10G066800 in soybean leaves under ACC

771 treatment examined by qRT-PCR. The data represent the mean $\pm \mathrm{SD}$ of three biological

772 replicates. The statistical significance was determined using Student's $t$-tests $(* * \mathrm{p}<0.01$,

$773 * \mathrm{p}<0.05, \mathrm{n}=3)$. SA: salicylates; JA: Jasmonic acid; ACC: ethylene precursor 1-

774 aminocyclopropane-1-carboxylicacid.

775 Table 1. Segmental duplications of trihelix paralogous pairs in soybean and inference of 776 duplication time.

777 Table 2. The differently expressed soybean trihelix genes at 4 time points after submergence

778 treatment identified by analyzing the public-RNA seq data.

779

780 Supplementary materials:

781 Figure S1. Multiple sequence alignment of GT domains of soybean and Arabidopsis trihelix

782 transcription factors. Helices 1, 2 and 3 represent trihelix structure identified in the GT domain.

783 Green, Yellow and blue shades indicate conserved amino acids.

784 Figure S2. Multi level consensus sequence and their logo of soybean trihelix proteins as

785 predicted by MEME program.

786 Figure S3. The average number of the exons in different trihelix sub-families.

787 Figure S4. The percentage of the cis-elements related to light, abiotic stresses (drought,

788 anaerobic and low-temperature) and plant hormones (abscisic acid, methyl jasomonate, ethylene 789 and salicylic acid).

790 Figure S5. qRT-PCR validation of the expression of 15 DEGs (differentially expressed genes)

791 responsive to submergence treatment at any time point. The data represent the mean $\pm \mathrm{SD}$ of

Peer] reviewing PDF | (2019:08:40490:3:0:NEW 29 Jan 2020) 
792 three biological replicates. The statistical significance was determined using Student's $t$-tests $(* *$ $793 \mathrm{p}<0.01, * \mathrm{p}<0.05, \mathrm{n}=3)$.

794

795 Figure S6. The expression profile of the 13 duplicated trihelix genes in the different tissues of 796 soybean. The genes pairs in red colour donated that they had distinct expression pattern. The 797 expression data was obtained from the public RNA-seq data published in Phytozome v12.1 798 (Libault et al., 2010; Goodstein et al., 2011).

799 Table S1. Information of trihelix family genes identified in the soybean genome.

800 Table S2. The FPKM (Fragments per kilobase for a million reads) value of soybean trihelix 801 genes in different tissues.

802 Table S3. Stress-related elements in the promoters of soybean Trihelix genes.

803 Table S4. putative MAPK phosphorylation sites of soybean trihelix genes.

804 Table S5. List of soybean trihelix genes with putative miRNA target sites.

805 Table S6. The FPKM value of the 71 trihelix genes 3 hours, 6 hours, 12 hours and 24 hours after 806 submergence treatment.

807 Table S7. Differentially expressed trihelix genes in soybean plants after submergence treatment.

808 Table S8. The expression of trihelix genes in the leaves of soybean under MAMP mixture 809 treatment.

810 Table S9. Sequences of primers used in this study. 
Figure 1

Figure 1. Chromosomal locations of soybean trihelix genes.

Gray bars represent chromosomes. Chromosome numbers are shown at the tops of the bar.

Trihelix genes are labeled at the right of the chromosomes. Scale bar on the left denotes the chromosome lengths (Mb). Chr: Chromosome. A-S: 20 chromosomes.

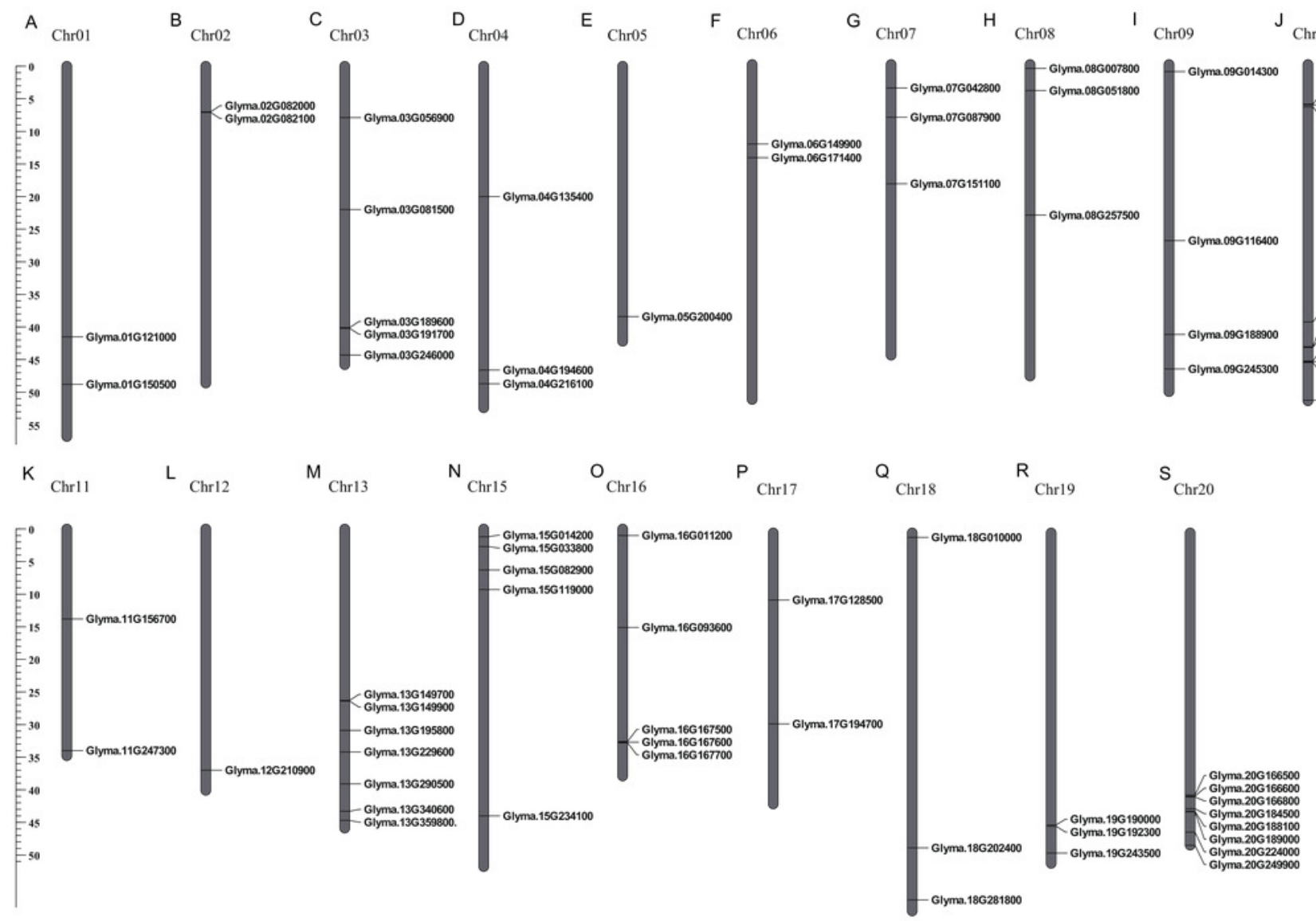


Figure 2

Figure 2. Circle plot of soybean chromosomes and the 26 trihelix family genes displayed as segmental duplicated gene pairs.

The thick green lines indicate duplicated trihelix gene pairs. Scale bar marked on the chromosome indicating chromosome lengths (Mb). Chr: Chromosome. 


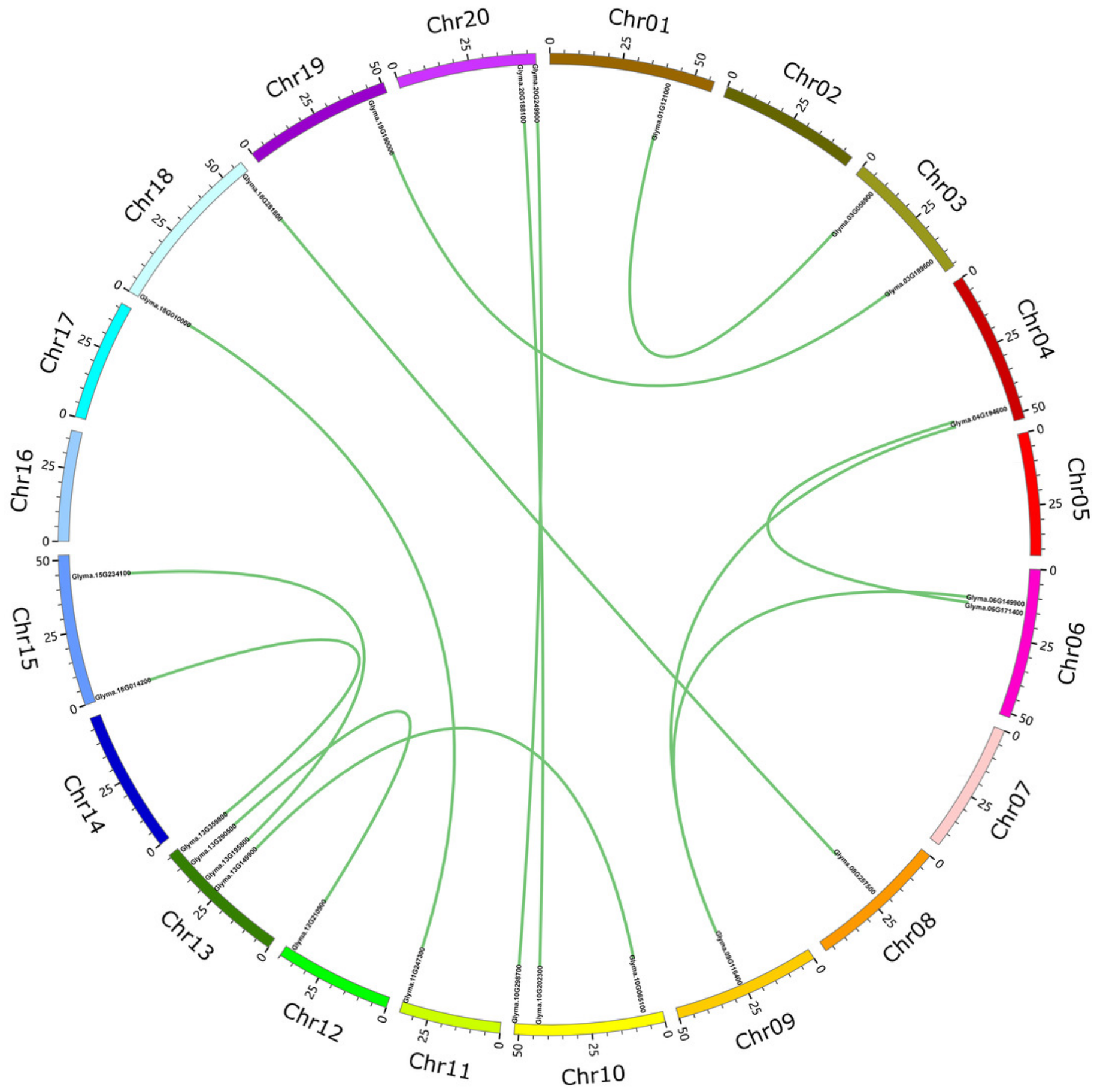


Figure 3

Figure 3. Phylogenetic tree based on protein sequences of trihelix genes in soybean, Arabidopsis thaliana, rice and tomato.

Gm, Glycine max; AT, Arabidopsis thaliana; Os, Oryza sativa; Solyc, Solanum lycopersicon. The Phylogenetic tree was constructed using the neighbor-joining (NJ) method, with 1000 bootstrap replicates, using MEGA v.7.0. 


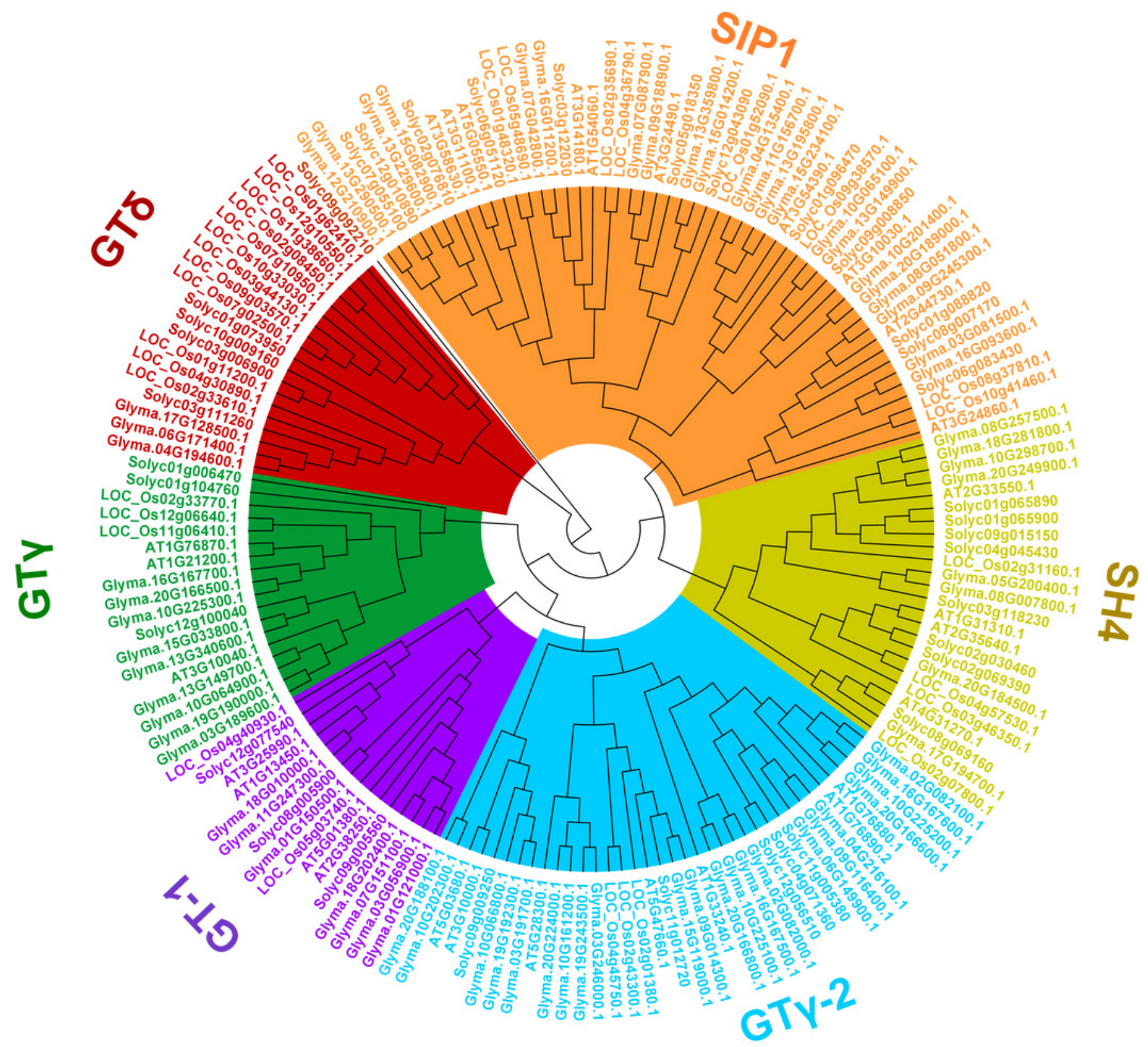




\section{Figure 4}

Figure 4. The conserved motifs and gene structure of the soybean trihelix family.

The genes in 6 sub-families were marked with different colors. (A) The conserved motifs of the soybean trihelix family. The boxes with different colors on the right denote 10 motifs. A detailed motif introduction is shown in Figure S2. The scale bar at the bottom indicates the lengths of the trihelix protein sequences. (B) Gene structures of the soybean trihelix family. CDS (coding sequence), introns, and untranslated regions (UTRs) are marked by yellow boxes, black lines, and green boxes, respectively. The sizes of exons and introns can be estimated using the scale at the bottom. 
A

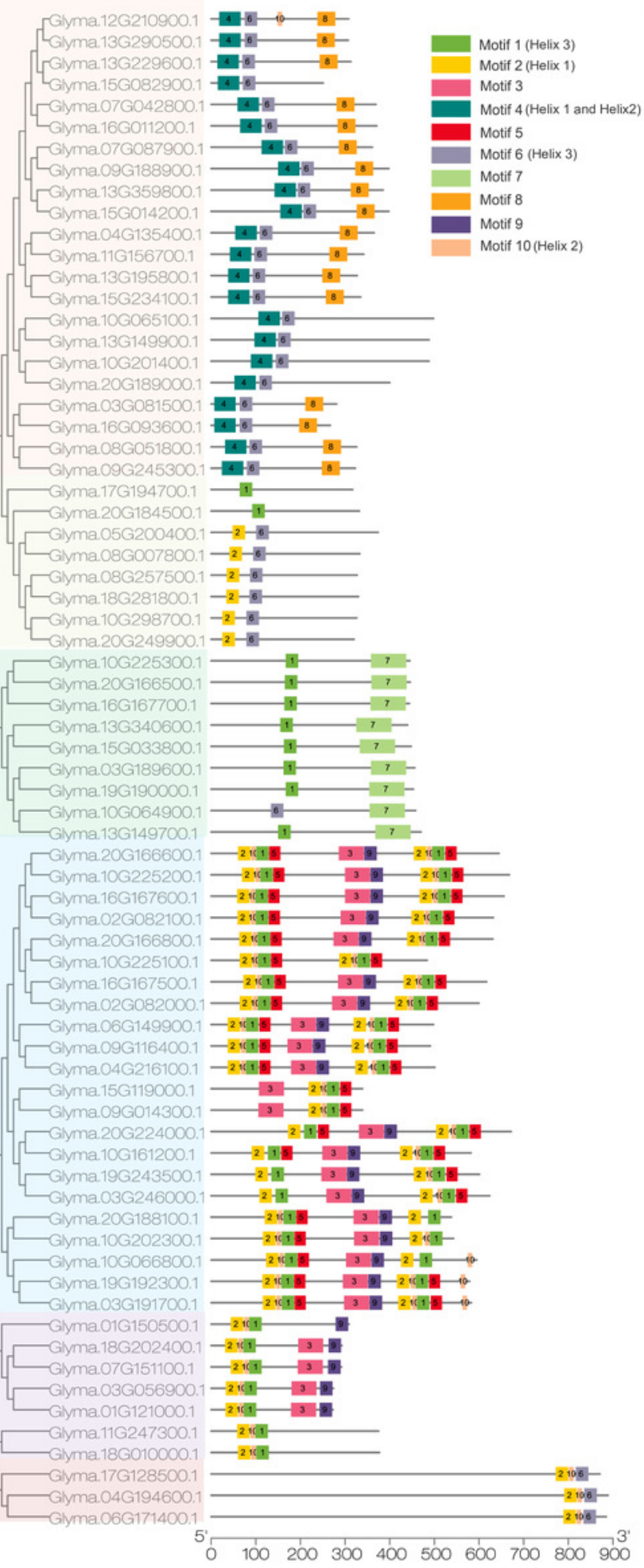

B

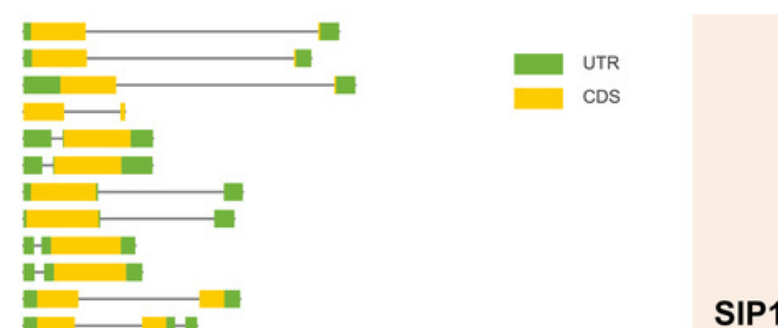

SIP1

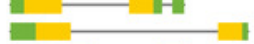

는

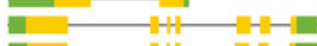

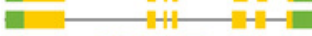

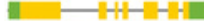

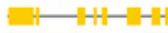

둔

(1)

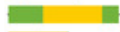

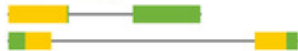

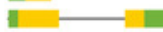

1 H

늘

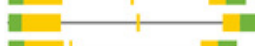

눈

in

두담

-

마믄

달

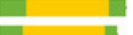

Eris

in

마뭄

-

믄

는

를- -

는 -

-

-

마늘

마는

분ㄷㄴ

드는

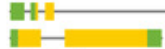

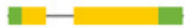

는

1 -

1

는

-

둔

-

-

는

대는

-

照

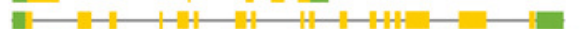

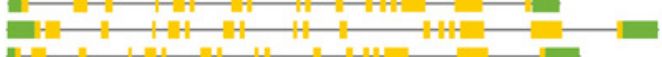

II-

\begin{tabular}{lllllll}
\hline & 2000 & 4000 & 6000 & 8000 & 10000 & 12000
\end{tabular} 


\section{Figure 5}

Figure 5. Heatmap of the expression profiles of the soybean trihelix genes in different tissues.

The expression values (Fragments per kilobase for a million reads, FPKM) for each gene in different tissues were log2 transformed before generating heat map. SAM: Shoot Apical Meristem. The expression data was obtained from the public RNA-seq data published in Phytozome v12.1 (Libault et al., 2010; Goodstein et al., 2011). 


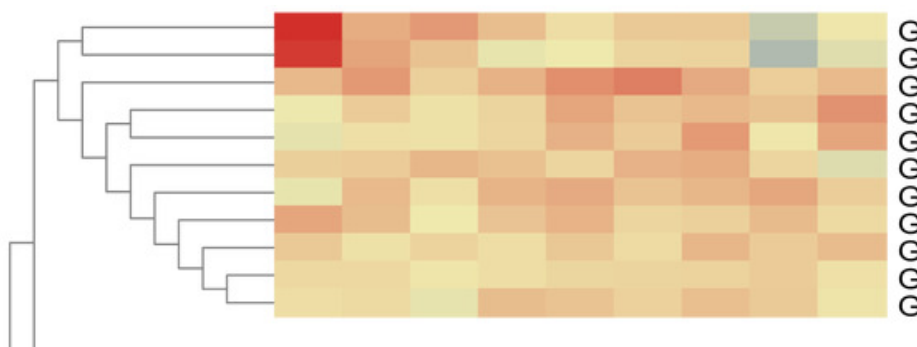

Glyma.10G225100

Glyma.20G166800

Glyma.16G167600

Glyma.07G042800

Glyma.16G011200

Glyma.20G166500

Glyma.08G051800

Group 1

Glyma.09G245300

Glyma.11G247300

Glyma.07G087900

Glyma.09G188900

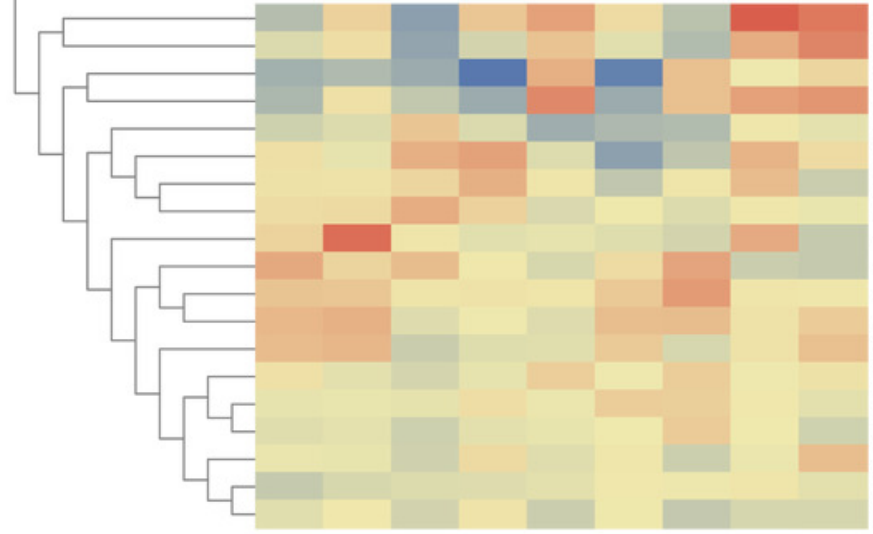

Glyma.10G161200

Glyma.20G224000

Glyma.03G246000

Glyma.19G243500

Glyma.08G257500

Glyma.15G234100

Glyma.13G195800

Glyma.20G249900

Glyma.02G082100

Glyma.10G225300

Group 2

Glyma.13G229600

Glyma.20G166600

Glyma.10G225200

Glyma.18G010000

Glyma.15G082900

Glyma.10G201400

Glyma.16G093600

Glyma.13G149900

Glyma.03G081500

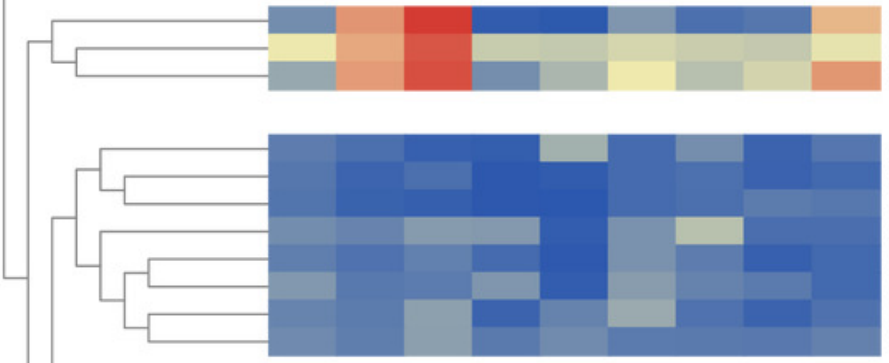

Glyma.03G189600 Glyma.10G298700

Glyma.13G149700

Group 3

Glyma.20G184500 Glyma.08G007800

Glyma.16G167700

Glyma.03G056900

Glyma.01G121000

Glyma.18G202400

Glyma.05G200400

Glyma.09G014300

Group 4

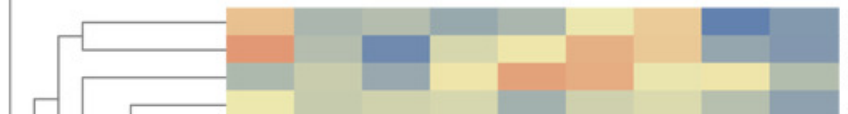

Glyma.06G149900

Glyma.16G167500

Glyma.06G171400

Glyma.01G150500

Glyma.15G033800

Glyma.10G065100

Glyma.17G194700

Glyma.13G340600

Glyma.13G359800

Glyma.15G014200

Glyma.04G194600

Glyma.02G082000

Glyma.04G216100

Glyma.17G128500

Glyma.09G116400

Group 5

Glyma.18G281800

Glyma.20G189000

Glyma.12G210900

Glyma.13G290500

Glyma.19G190000

Glyma.10G064900

Glyma.15G119000

Glyma.10G066800

Glyma.20G188100

Glyma.07G151100

Glyma.04G135400

Glyma.11G156700

Glyma.10G202300

Glyma.03G191700

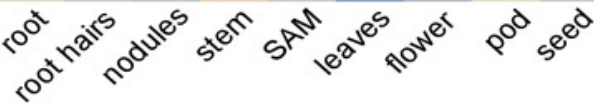

Glyma.19G192300

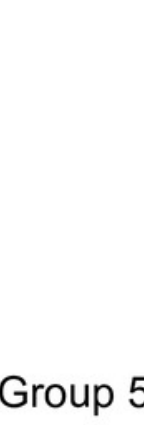




\section{Figure 6}

Figure 6. Predicted cis-elements in the promoter regions ( $1500 \mathrm{bp}$ upstream from the transcription initiation site) of the soybean trihelix genes.

The scale bar at the bottom indicates the length of promoter sequence. ABRE: abscisic acid responsive element; ARE: anaerobic responsive element; DRE: drought responsive element; ERE: ethylene responsive element; LTR: low temperature responsive element; MBS: drought responsive element; TCA-element: salicylic acid responsive element; TGACG-motif: methyl jasomonate responsive element. 
Glyma.01G121000 Glyma.01G150500

Glyma.02G082000

Glyma.02G082100 Glyma.03G056900 Glyma.03G081500 Glyma.03G189600 Glyma.03G191700 Glyma.03G246000

Glyma.04G135400 Glyma.04G194600 Glyma.04G216100 Glyma.05G200400 Glyma.06G149900 Glyma.06G171400 Glyma.07G042800 Glyma.07G087900 Glyma.07G151100 Glyma.08G007800 Glyma.08G051800 Glyma.08G257500 Glyma.09G014300 Glyma.09G116400 Glyma.09G188900 Glyma.09G245300 Glyma.10G064900 Glyma.10G065100 Glyma.10G066800 Glyma.10G161200 Glyma.10G201400 Glyma.10G202300 Glyma.10G225100 Glyma.10G225200 Glyma.10G225300 Glyma.10G298700 Glyma.11G156700 Glyma.11G247300 Glyma. 12G210900 Glyma.13G149700 Glyma.13G149900 Glyma.13G195800 Glyma.13G229600 Glyma.13G290500 Glyma.13G340600 Glyma.13G359800 Glyma.15G014200 Glyma. $15 \mathrm{G} 033800$ Glyma.15G082900 Glyma.15G119000 Glyma. $15 \mathrm{G} 234100$ Glyma.16G011200 Glyma.16G093600 Glyma.16G167500 Glyma.16G167600 Glyma.16G167700 Glyma. $17 \mathrm{G} 128500$ Glyma.17G194700 Glyma.18G010000 Glyma.18G202400 Glyma.18G281800 Glyma.19G190000 Glyma.19G192300 Glyma.19G243500 Glyma.20G166500 Glyma.20G166600 Glyma.20G166800 Glyma.20G184500 Glyma.20G188100 Glyma.20G189000 Glyma.20G224000 Glyma.20G249900

(s)

Legend:

Exon $\mathrm{ABRE}$

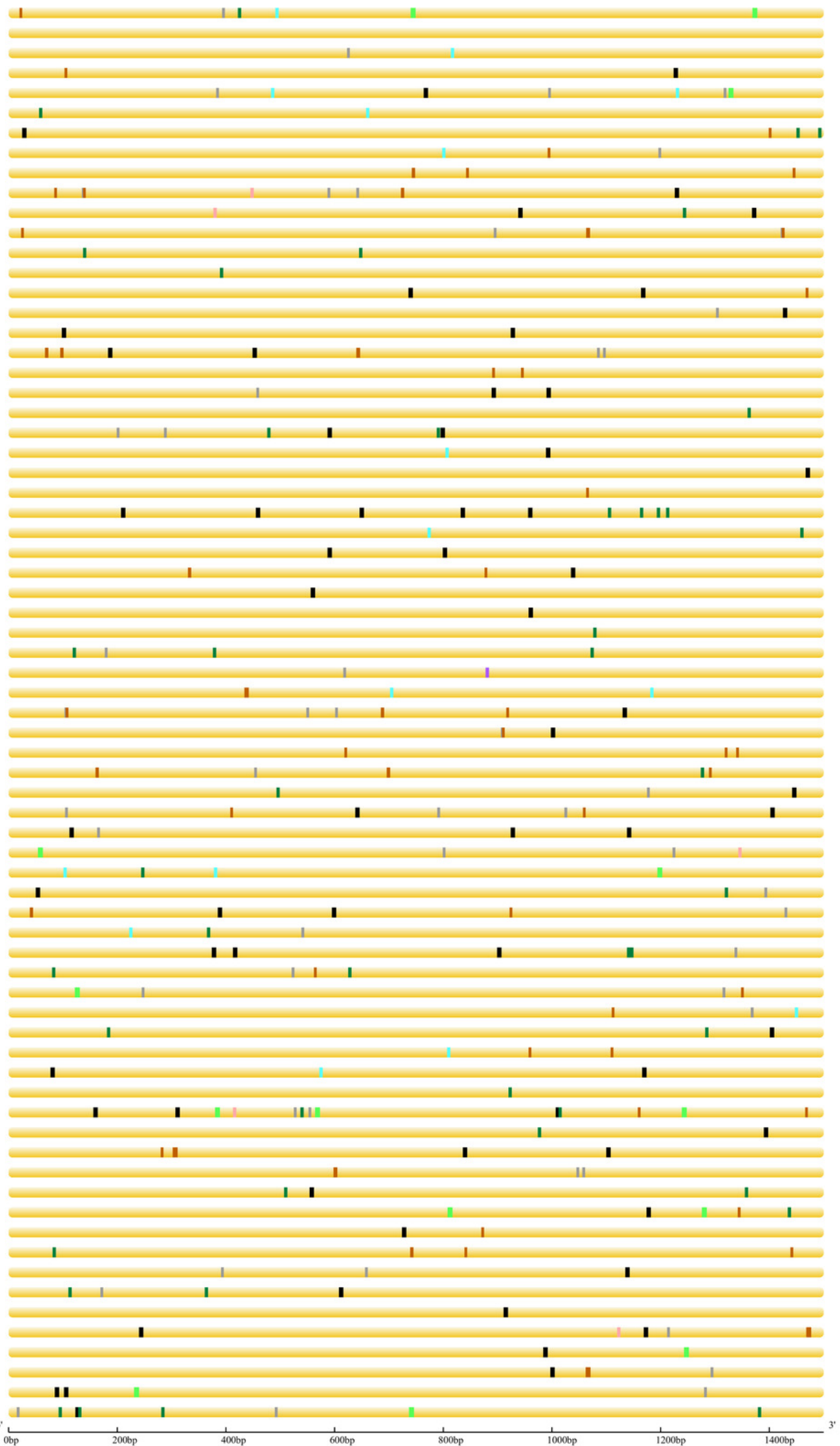

ARE $\square$ DRE $\square$ LTR $\square$ MBS $\square$ TCA-element 


\section{Figure 7}

Figure 7. Heatmap of the differentially expressed trihelix genes of soybean suffering from submergence treatment.

The RNA-seq data was obtained from NCBI Sequence Read Archive (SRA) with the accession number: SRP181976 (Lin et al., 2019). The expression values (Fragments per kilobase for a million reads, FPKM) for each gene were log2 transformed before generating the heatmap. ck-3h, ck-6h, ck-12h and ck-24h indicate the roots from plants untreated by submergence stress; F-3h, F-6h, F-12h, F-24h denote the roots sampled at $3 \mathrm{~h}, 6 \mathrm{~h}, 12 \mathrm{~h}$ and $24 \mathrm{~h}$ after submergence stress treatment, respectively. 


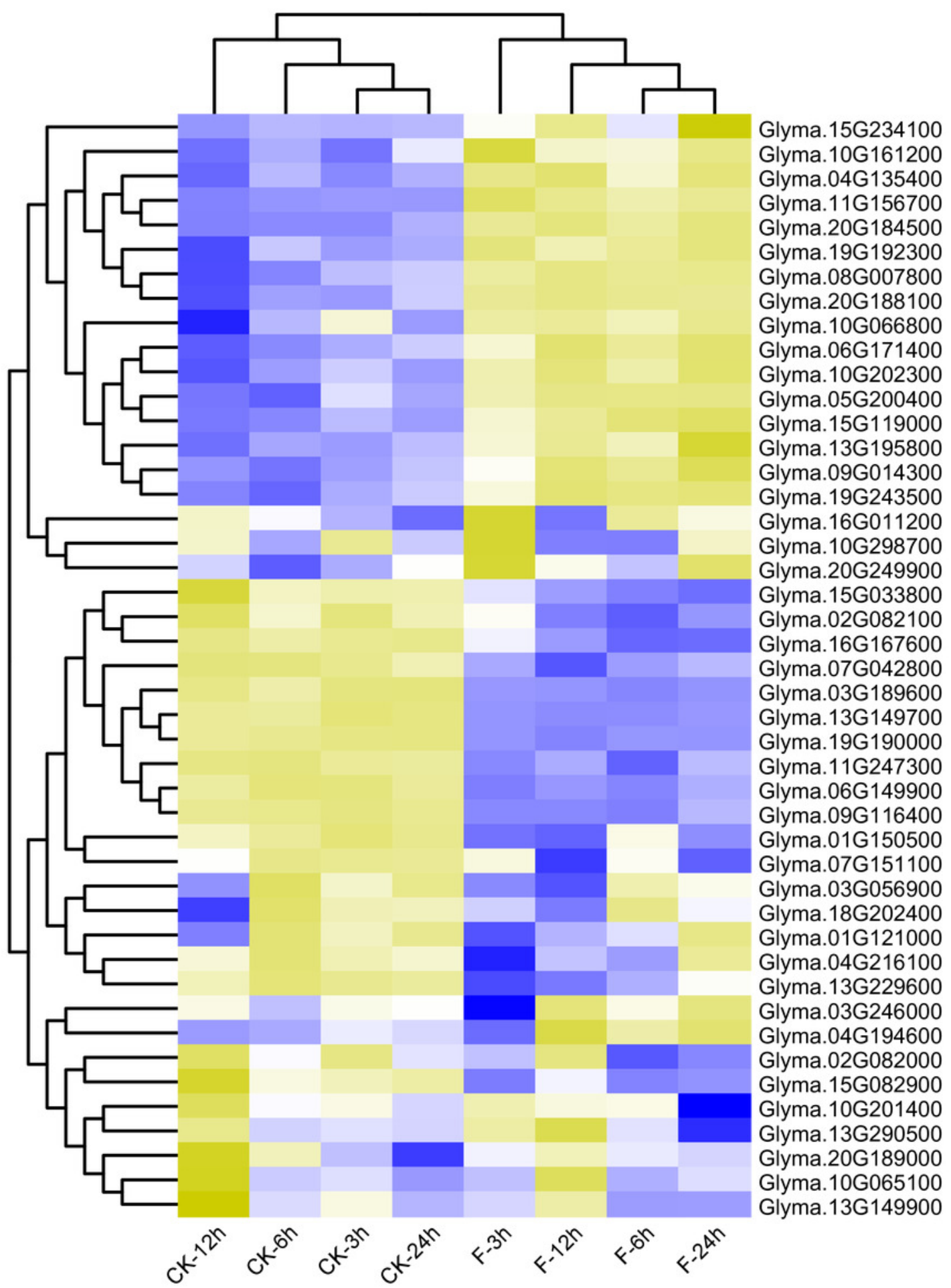




\section{Figure 8}

Figure 8. The expression profiles of 12 selected trihelix genes under $\mathrm{NaCl}$ and $\mathrm{ABA}$ treatment examined by qRT-PCR.

A-L: The relative expression levels of 12 selected trihelix genes under $\mathrm{NaCl}$ treatment. $0 \mathrm{~h}, 3$

$\mathrm{h}, 6 \mathrm{~h}$, and $12 \mathrm{~h}$ denoted the roots sampled $0 \mathrm{~h}, 3 \mathrm{~h}, 6 \mathrm{~h}$, and $12 \mathrm{~h}$ after $100 \mathrm{mM} \mathrm{NaCl}$ treatment, respectively. The data represent the mean \pm SD of three biological replicates. The statistical significance was determined using Student's t-tests ( ${ }^{* *} p<0.01,{ }^{*} p<0.05, n=3$ ); M$\mathrm{X}$ : The relative expression levels of 12 selected trihelix genes under ABA treatment. $0 \mathrm{~h}, 3 \mathrm{~h}$, $6 \mathrm{~h}$, and $12 \mathrm{~h}$ denoted the roots sampled $0 \mathrm{~h}, 3 \mathrm{~h}, 6 \mathrm{~h}$, and $12 \mathrm{~h}$ after $100 \mu \mathrm{M}$ ABA treatment, respectively. The data represent the mean \pm SD of three biological replicates. The statistical significance was determined using Student's t-tests ( $* * p<0.01, * P<0.05, n=3$ ). 


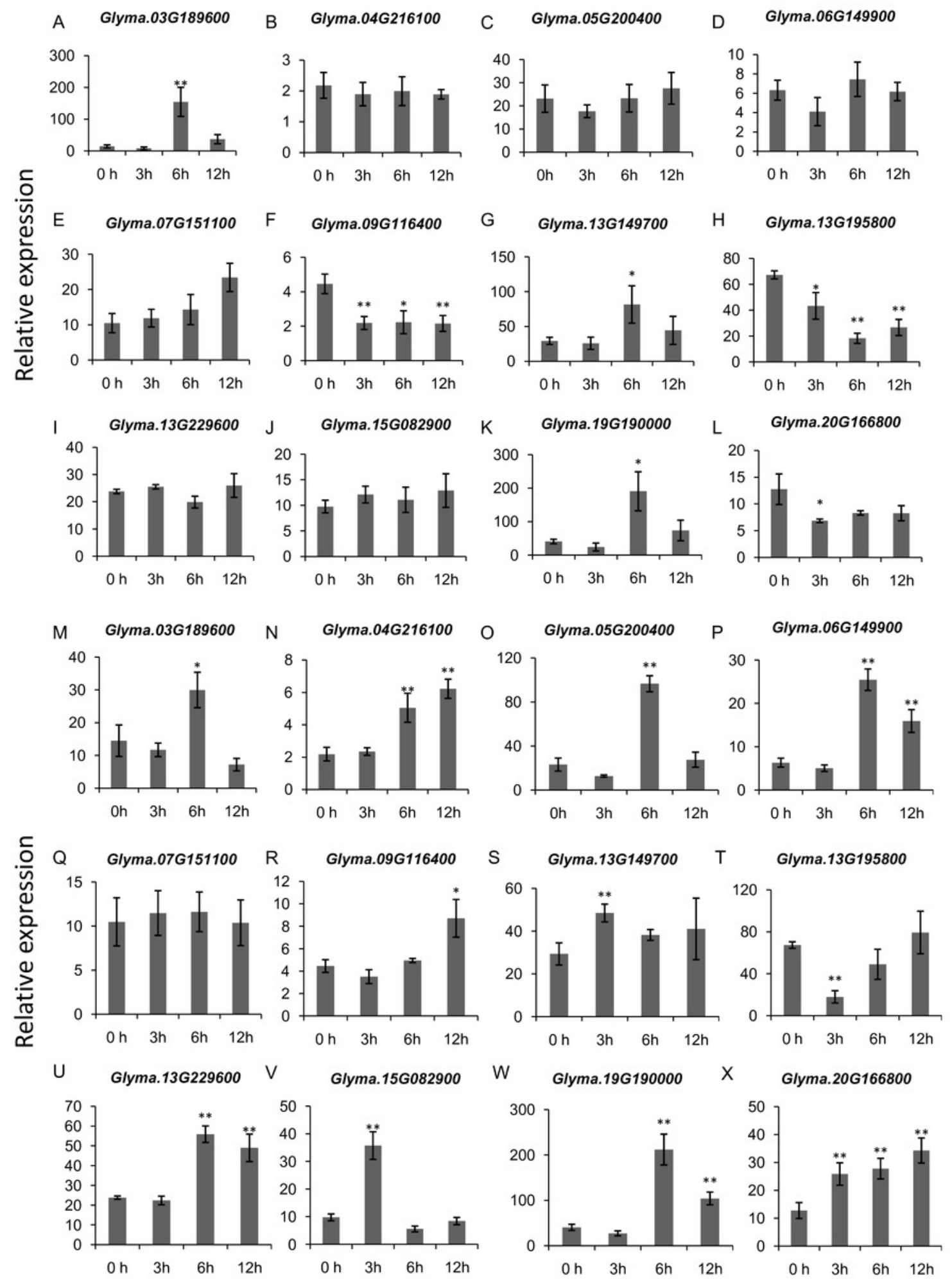




\section{Figure 9}

Figure 9. The DEGs (differentially expressed genes) of soybean trihelix genes under MAMP (microbe-associated molecular patterns) mixtures treatment and their expression patterns under SA, JA and ACC treatment.

A: 2 DEGs (Glyma.01G121000 and Glyma.10G066800) in soybean leaves under MAMP (mixture with flg22 and chintin) treatment for $30 \mathrm{~min}$. The microarray data was obtained from GEO series accession no. GSE32642 (Valdés-López et al., 2011). B-C: The expression pattern of Glyma.01G121000 and Glyma.10G066800 in soybean leaves under SA treatment examined by qRT-PCR. D-E: The expression pattern of Glyma.01G121000 and Glyma.10G066800 in soybean leaves under JA treatment examined by qRT-PCR; F-G: The expression pattern of Glyma.01G121000 and Glyma.10G066800 in soybean leaves under ACC treatment examined by qRT-PCR. The data represent the mean \pm SD of three biological replicates. The statistical significance was determined using Student's $t$-tests ( $* * p<0.01$, $\left.{ }^{*} p<0.05, n=3\right)$. SA: salicylates; JA: Jasmonic acid; ACC: ethylene precursor 1aminocyclopropane-1-carboxylicacid. 
A

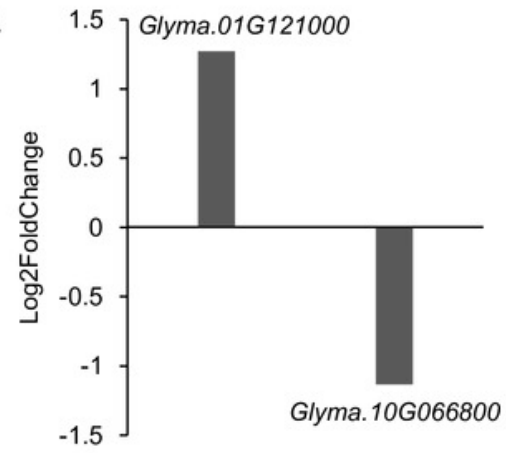

D

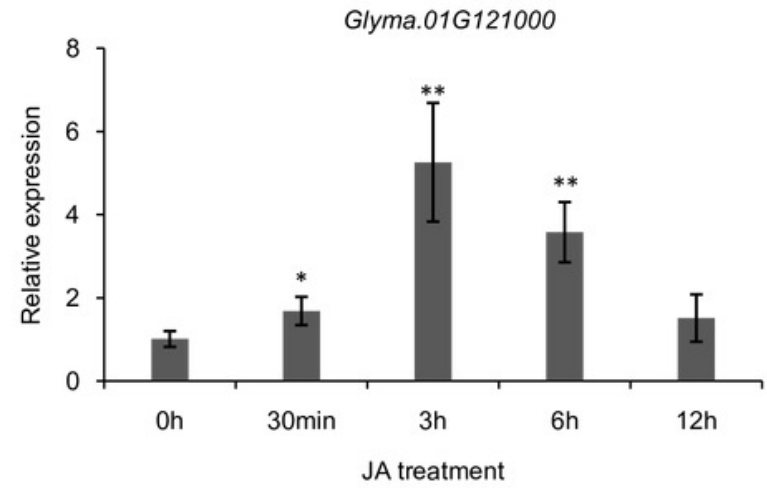

$\mathbf{F}$

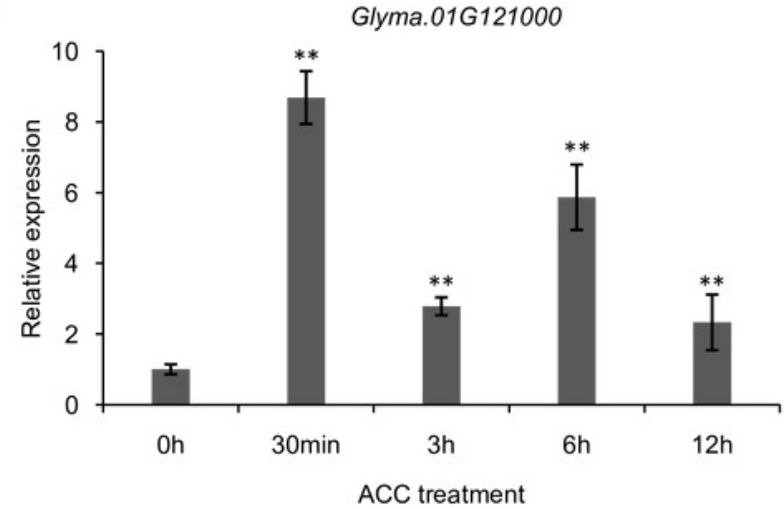

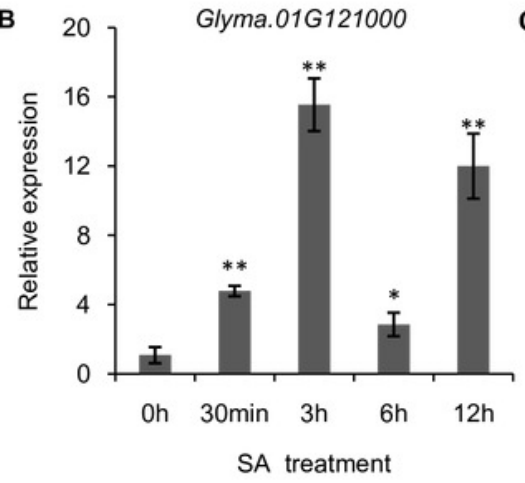

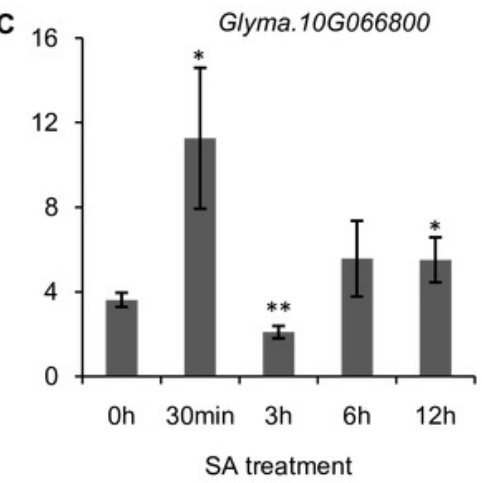

E

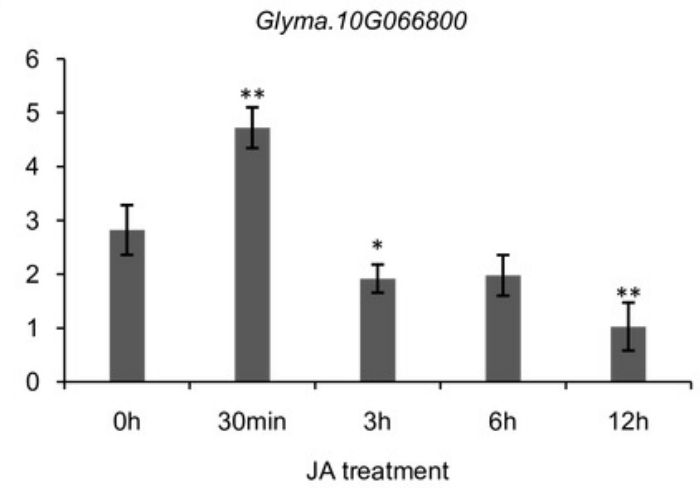

G

Glyma.10G066800

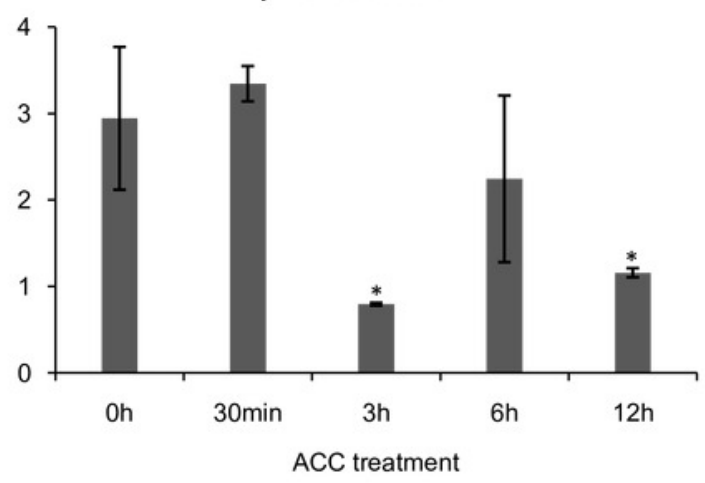




\section{Table $\mathbf{1}$ (on next page)}

Table 1. Segmental duplications of trihelix paralogous pairs in soybean and inference of duplication time. 
1 Table 1. Segmental duplications of trihelix paralogous pairs in soybean and inference of 2 duplication time

3

\begin{tabular}{|c|c|c|c|c|c|c|}
\hline Trihelix gene pairs & Ka & Ks & $\mathrm{Ka} / \mathrm{Ks}$ & $\begin{array}{l}\text { P-Value } \\
\text { (Fisher) }\end{array}$ & $\begin{array}{c}\text { Length } \\
\text { (bp) }\end{array}$ & $\begin{array}{c}\text { Approximate } \\
\text { duplication date } \\
\text { (MYA) }\end{array}$ \\
\hline $\begin{array}{l}\text { Glyma.01G121000 } \\
\& \text { Glyma.03G056900 }\end{array}$ & 0.0320 & 0.2021 & 0.1584 & $2.22 \mathrm{E}-11$ & 792 & 16.57 \\
\hline $\begin{array}{l}\text { Glyma.04G194600 } \\
\& \text { Glyma.06G171400 }\end{array}$ & 0.0156 & 0.1079 & 0.1445 & $2.20 \mathrm{E}-20$ & 2640 & 8.84 \\
\hline $\begin{array}{l}\text { Glyma.04G216100 } \\
\& \text { Glyma.09G116400 }\end{array}$ & 0.0084 & 0.0204 & 0.4141 & 0.041626 & 1467 & 1.67 \\
\hline $\begin{array}{l}\text { Glyma.08G257500 } \\
\& \text { Glyma.18G281800 }\end{array}$ & 0.0234 & 0.1620 & 0.1442 & $1.35 \mathrm{E}-12$ & 972 & 13.28 \\
\hline $\begin{array}{l}\text { Glyma.09G116400 } \\
\& \text { Glyma.06G149900 }\end{array}$ & 0.0432 & 0.1596 & 0.2705 & $8.73 \mathrm{E}-11$ & 1431 & 13.08 \\
\hline $\begin{array}{l}\text { Glyma.10G065100 } \\
\& \text { Glyma.13G149900 }\end{array}$ & 0.0176 & 0.1279 & 0.1376 & $8.36 \mathrm{E}-15$ & 1437 & 10.48 \\
\hline $\begin{array}{l}\text { Glyma.10G202300 } \\
\& \text { Glyma.20G188100 }\end{array}$ & 0.0364 & 0.1109 & 0.3286 & $2.45 \mathrm{E}-07$ & 1593 & 9.09 \\
\hline $\begin{array}{l}\text { Glyma.10G298700 } \\
\& \text { Glyma.20G249900 }\end{array}$ & 0.0537 & 0.2249 & 0.2388 & $1.95 \mathrm{E}-11$ & 945 & 18.43 \\
\hline $\begin{array}{l}\text { Glyma.11G247300 } \\
\& \text { Glyma.18G010000 }\end{array}$ & 0.0013 & 0.1009 & 0.0126 & 0 & 1119 & 8.27 \\
\hline $\begin{array}{l}\text { Glyma.12G210900 } \\
\& \text { Glyma.13G290500 }\end{array}$ & 0.0252 & 0.2416 & 0.1042 & $1.21 \mathrm{E}-16$ & 909 & 19.80 \\
\hline $\begin{array}{l}\text { Glyma.13G195800 } \\
\& \text { Glyma.15G234100 }\end{array}$ & 0.0600 & 0.2071 & 0.2898 & $9.48 \mathrm{E}-09$ & 957 & 16.97 \\
\hline $\begin{array}{l}\text { Glyma.13G359800 } \\
\& \text { Glyma.15G014200 }\end{array}$ & 0.0355 & 0.1386 & 0.2565 & $1.71 \mathrm{E}-07$ & 1146 & 11.36 \\
\hline $\begin{array}{l}\text { Glyma.19G190000 } \\
\text { \&Glyma.03G189600 }\end{array}$ & 0.0274 & 0.1288 & 0.2126 & $7.40 \mathrm{E}-11$ & 1320 & 10.55 \\
\hline
\end{tabular}

Note: Non-synonymous (Ka) and synonymous $(\mathrm{Ks})$ show the substitution rates. $\mathrm{Ka} / \mathrm{Ks}$ is the ratio of non-synonymous (Ka) versus 5 synonymous (Ks) mutations. This ratio is used as indicator to determine the selective pressure or strength on a protein-encoding 6 gene. $\mathrm{Ka} / \mathrm{Ks}=1$ shows "no selection", $\mathrm{Ka} / \mathrm{Ks}<1$ indicates "negative or purifying selection" and $\mathrm{Ka} / \mathrm{Ks}>1$ denotes "positive or 7 Darwinian selection'. 


\section{Table 2 (on next page)}

Table 2. The differently expressed soybean trihelix genes at 4 time points after submergence treatment identified by analyzing the public-RNA seq data. 
Table 2. The differently expressed soybean trihelix genes at 4 time points after submergence treatment identified by analyzing the public-RNA seq data

\begin{tabular}{|c|c|c|c|c|c|c|c|c|c|}
\hline Gene ID & clade & $\begin{array}{c}\log 2 F C \\
(3 h)\end{array}$ & $\begin{array}{c}\text { adjusted } \\
\text { pvalue } \\
(3 h)\end{array}$ & $\begin{array}{c}\log 2 F C \\
(6 h)\end{array}$ & $\begin{array}{c}\text { adjusted } \\
\text { pvalue } \\
\text { (6h) }\end{array}$ & $\begin{array}{c}\log 2 F C \\
(12 h)\end{array}$ & $\begin{array}{c}\text { adjusted } \\
\text { pvalue } \\
(12 \mathrm{~h})\end{array}$ & $\begin{array}{c}\log 2 F C \\
(24 h)\end{array}$ & $\begin{array}{c}\text { adjusted } \\
\text { pvalue } \\
\text { (24h) }\end{array}$ \\
\hline Glyma.13G229600 & SIP1 & 1.78 & $1.61 \mathrm{E}-30$ & 1.59 & $7.00 \mathrm{E}-21$ & 1.64 & $2.48 \mathrm{E}-09$ & 1.14 & $7.24 \mathrm{E}-14$ \\
\hline Glyma.11G156700 & SIP1 & -1.93 & $4.68 \mathrm{E}-11$ & -1.04 & 3.34E-04 & -1.46 & $9.01 \mathrm{E}-04$ & -1.26 & $1.10 \mathrm{E}-05$ \\
\hline Glyma.07G042800 & SIP1 & 1.69 & $9.01 \mathrm{E}-33$ & 1.94 & $7.20 \mathrm{E}-41$ & 2.43 & $2.10 \mathrm{E}-24$ & 1.54 & $1.47 \mathrm{E}-16$ \\
\hline Glyma.15G082900 & SIP1 & 1.31 & $2.51 \mathrm{E}-17$ & 1.26 & $3.32 \mathrm{E}-14$ & 1.6 & $5.60 \mathrm{E}-09$ & 1.47 & $7.56 \mathrm{E}-12$ \\
\hline Glyma.03G189600 & $\mathrm{GT} \gamma$ & 10.27 & $8.06 \mathrm{E}-161$ & 7.83 & $8.13 E-49$ & 9.76 & $1.56 \mathrm{E}-286$ & 10.87 & $1.04 \mathrm{E}-142$ \\
\hline Glyma.13G149700 & $\mathrm{GT} \gamma$ & 7.99 & $6.01 \mathrm{E}-278$ & 6.78 & $2.31 \mathrm{E}-67$ & 7.12 & $3.91 \mathrm{E}-174$ & 7.68 & $9.37 \mathrm{E}-287$ \\
\hline Glyma.19G190000 & GT $\gamma$ & 8.66 & $4.32 \mathrm{E}-232$ & 8.15 & $1.89 \mathrm{E}-83$ & 8.57 & $0.00 \mathrm{E}+00$ & 8.94 & $6.04 \mathrm{E}-146$ \\
\hline Glyma.07G151100 & GT-1 & 1.03 & $4.18 \mathrm{E}-03$ & 1.29 & $3.58 \mathrm{E}-06$ & 1.8 & $9.12 \mathrm{E}-06$ & 2.14 & $1.08 \mathrm{E}-12$ \\
\hline Glyma.11G247300 & GT-1 & 1.53 & $7.30 \mathrm{E}-16$ & 1.91 & $5.00 \mathrm{E}-19$ & 1.68 & $8.41 \mathrm{E}-14$ & 1.45 & $1.39 \mathrm{E}-10$ \\
\hline Glyma.16G167600 & GT-2 & 1.29 & $2.63 \mathrm{E}-11$ & 2.02 & $1.35 \mathrm{E}-23$ & 2.04 & $2.25 \mathrm{E}-14$ & 2.23 & $2.32 \mathrm{E}-19$ \\
\hline Glyma.06G149900 & GT-2 & 3.14 & $2.06 \mathrm{E}-59$ & 3.23 & $6.49 \mathrm{E}-105$ & 2.73 & 2.54E-19 & 2.54 & $4.74 \mathrm{E}-23$ \\
\hline Glyma.20G224000 & GT-2 & -2.19 & $2.22 \mathrm{E}-05$ & -1.5 & $2.54 \mathrm{E}-03$ & -1.67 & $2.92 \mathrm{E}-02$ & -2.06 & 4.93E-05 \\
\hline Glyma.09G116400 & GT-2 & 4.04 & $7.48 \mathrm{E}-90$ & 4.06 & $5.68 \mathrm{E}-107$ & 3.94 & $2.36 \mathrm{E}-38$ & 3.32 & $6.56 \mathrm{E}-40$ \\
\hline Glyma.05G200400 & SH4 & -1.43 & $1.33 \mathrm{E}-02$ & -5.29 & $2.74 \mathrm{E}-08$ & -5.72 & $1.11 \mathrm{E}-09$ & -5.36 & 2.72E-08 \\
\hline Glyma.08G007800 & SH4 & -1.92 & $2.31 \mathrm{E}-02$ & -2.72 & $5.30 \mathrm{E}-03$ & -4.45 & $8.90 \mathrm{E}-06$ & -2.74 & $6.54 \mathrm{E}-03$ \\
\hline
\end{tabular}

